\title{
Imposing virtual origins on the velocity components in direct numerical simulations
}

\author{
Garazi Gómez-de-Segura ${ }^{1}$, Ricardo García-Mayoral ${ }^{1, *}$ \\ ${ }^{a}$ Department of Engineering, University of Cambridge, Trumpington St, Cambridge CB2 1PZ, UK
}

\begin{abstract}
The relative wall-normal displacement of the origin perceived by different components of near-wall turbulence is known to produce a change in drag. This effect is produced for instance by drag-reducing surfaces of small texturesize like riblets and superhydrophobic surfaces. To facilitate the research on how these displacements alter near-wall turbulence, this paper studies different strategies to model such displacement effect through manipulated boundary conditions. Previous research has considered the effect of offsetting the virtual origins perceived by the tangential components of the velocity from the reference, boundary plane, where the wall-normal velocity was set to zero. These virtual origins are typically characterised by slip-length coefficients in Robin, slip-like boundary conditions. In this paper, we extend this idea and explore several techniques to define and implement virtual origins for all three velocity components on direct numerical simulations (DNSs) of channel flows, with special emphasis on the wall-normal velocity. The aim of this work is to provide a suitable foundation to extend the existing understanding on how these virtual origins affect the near-wall turbulence, and ultimately aid in the formulation of simplified models that capture the effect of complex surfaces on the overlying flow and on drag, without the need to resolve fully the turbulence and the surface texture. From the techniques tested, Robin boundary conditions for all three velocities are found to be the most satisfactory method to impose virtual origins, relating the velocity components to their respective wall-normal gradients linearly. Our results suggest that the effect of virtual origins on the flow, and hence the change in drag that they produce, can be reduced to an offset between the virtual origin perceived by the mean flow and that perceived by the overlying turbulence, and that turbulence remains otherwise smooth-wall-like, as proposed by Luchini (1996). The origin for turbulence, however, would not be set by the spanwise virtual origin alone, but by a combination of the spanwise and wall-normal origins. These observations suggest the need for an extension of Luchini's virtual-origin theory to predict the change in drag, accounting for the wall-normal transpiration when its effect is not negligible.
\end{abstract}

Keywords:

\section{Introduction}

Many passive turbulent drag reduction techniques rely on the ability of small surface textures to manipulate the overlying near-wall cycle and change the skin friction drag. Examples of such surfaces include riblets (Walsh and Lindemann, 1984; García-Mayoral and Jiménez, 2011), superhydrophobic surfaces (Rothstein, 2010), or anisotropic permeable substrates (Gómez-deSegura and García-Mayoral, 2019). Solving the flow over these surfaces is computationally expensive, as the

\footnotetext{
${ }^{*}$ Corresponding author

Email addresses: ggomezdesegura@protonmail .com (Garazi Gómez-de-Segura), r.gmayoral@eng. cam . ac.uk (Ricardo García-Mayoral)
}

Preprint submitted to International Journal of Heat and Fluid Flow resolution required to resolve simultaneously the turbulence dynamics and the detail of the texture can be prohibitively high. It is therefore of interest to find simplified models that capture the effect that such surfaces produce on the overlying flow, with particular emphasis on predicting surface drag.

It has previously been proposed that small textures alter drag by offsetting the apparent origins -the wallnormal location where the presence of a smooth wall is perceived- experienced by the mean flow and the nearwall turbulent structures, and that these 'virtual' origins are those perceived by the streamwise and spanwise components of the velocity, respectively (Luchini et al., 1991; Luchini, 1996). More recently, it has been suggested that the virtual origin for turbulence was not de-

August 6, 2020 
termined solely by the origin perceived by the spanwise velocity, but by an interplay between the latter and the origin perceived by the wall-normal velocity (Fairhall and García-Mayoral, 2018). To provide the means to investigate this phenomenon, the present paper aims to investigate the suitability of different techniques to impose virtual origins on the three velocity components independently. The focus is on how the overlying flow responds to these apparent origins, and not how any particular surface can produce this effect. We refer the reader to the literature on homogenisation techniques (Bottaro, 2019; Lācis et al., 2020) for details on how consistent effective boundary conditions can be derived for specific textures.

García-Mayoral et al. (2019) have recently reviewed the drag-altering effect of textured surfaces. When the texture is small compared to the overlying turbulent structures, its direct effect is confined to the immediate vicinity of the surface, while farther away from the wall its effect is reflected merely in a shift of the velocity profile, $\Delta U^{+}$(Clauser, 1956). The superscript ' + ' indicates scaling in viscous units, defined in terms of the kinematic viscosity $v$ and the friction velocity $u_{\tau}=\sqrt{\tau_{w}}$, where $\tau_{w}$ is the kinematic shear stress at the wall. The mean velocity in the logarithmic region can then be expressed as $U^{+}=1 / \kappa \log y^{+}+B+\Delta U^{+}$, where $y$ is the wall-normal coordinate, $\kappa$ is the Kármán constant and $B$ is the smooth-wall logarithmic intercept. Note that in this paper we take $\Delta U^{+}$as positive when the mean velocity $U^{+}$increases, contrary to roughness literature, where it takes the opposite sign (Chung et al., 2021). The shift $\Delta U^{+}$can be used as an essentially Reynolds-number-independent measure of the change in drag (García-Mayoral and Jiménez, 2011; Spalart and McLean, 2011; Gatti and Quadrio, 2016; GarcíaMayoral et al., 2019). If $\Delta U^{+}>0$, the logarithmic region is shifted upwards and drag is reduced. Conversely, if $\Delta U^{+}<0$, the logarithmic region is shifted downwards and drag is increased.

The studies on how complex surfaces affect the overlying turbulent flow and create this shift $\Delta U^{+}$date back to the early 90s. The first studies were on riblets (Luchini et al., 1991), but the same mechanism applies also to other complex surfaces. When the surface texture is small, the overlying flow does not perceive the detail of the texture, but a homogenised effect of it, and the velocity components may appear to vanish at different heights. Taking the outermost plane of the surface geometry as reference, $y^{+}=0$, Luchini et al. (1991) proposed that the change in drag depends on the offset between a streamwise and a spanwise 'protrusion heights'. Luchini (1996) suggested that these heights (a)

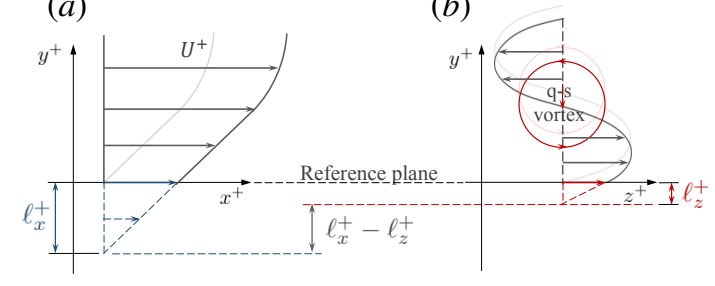

Figure 1: Sketch of the $(a)$ streamwise and $(b)$ spanwise slip lengths, $\ell_{x}^{+}$and $\ell_{z}^{+}$, and the corresponding virtual origins at $y^{+}=-\ell_{x}^{+}$and $y^{+}=-\ell_{z}^{+}$. A quasi-streamwise vortex (q-s vortex), inducing crossflow $w^{+}$, is sketched in $(b)$.

would correspond to the virtual, equivalent smooth wall perceived by the mean flow, at $y^{+}=-\ell_{x}^{+}$, and that perceived by the turbulent fluctuations, particularly the quasi-streamwise vortices of the near-wall cycle, and that turbulence would remain otherwise as that over a smooth wall. Given that near the surface the quasistreamwise vortices induce primarily a transverse shear in the spanwise direction, Luchini (1996) proposed that the virtual origin of turbulence is given by that of the spanwise velocity, $y^{+}=-\ell_{z}^{+}$. Drag reduction over riblets, and by extension over any non-smooth surface (Jiménez, 1994), could then be viewed as a virtualorigin effect, as depicted in figure 1, such that

$$
\Delta U^{+} \propto \ell_{x}^{+}-\ell_{z}^{+} .
$$

If $\ell_{z}^{+}<\ell_{x}^{+}$, the surface texture would impede the penetration of turbulence as deep as the mean flow, and quasi-streamwise vortices would be comparatively 'pushed' upwards, away from the origin for the mean flow, $y^{+}=-\ell_{x}^{+}$. As a result, the local turbulence mixing close to the surface would decrease, thereby reducing the shear and the skin friction (Orlandi and Jiménez, 1994). Conversely, if $\ell_{x}^{+}<\ell_{z}^{+}$, the vortices would perceive a deeper origin than the mean flow and friction drag would increase. This suggests that the effect of complex surfaces can be characterised by streamwise and spanwise Robin slip conditions at a reference plane $y=0$, as portrayed in figure 1 ,

$$
\begin{aligned}
& \left.u^{+}\right|_{y^{+}=0}=\left.\ell_{x}^{+} \frac{\partial u^{+}}{\partial y}\right|_{y^{+}=0}, \\
& \left.w^{+}\right|_{y^{+}=0}=\left.\ell_{z}^{+} \frac{\partial w^{+}}{\partial y}\right|_{y^{+}=0},
\end{aligned}
$$

where the lengths $\ell_{x}^{+}$and $\ell_{z}^{+}$would characterise the virtual origins for the streamwise and spanwise velocities, $u^{+}$and $w^{+}$. These lengths are also called 'slip lengths' in the superhydrophobic community, as well as 'protrusion heights' in the riblet community. 


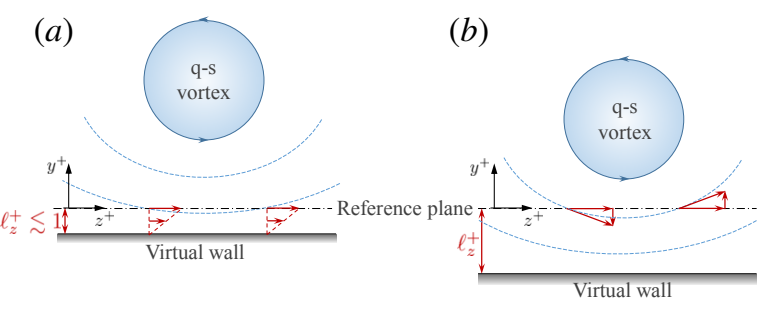

Figure 2: Schematic of the spanwise and wall-normal velocities induced by quasi-streamwise vortices at the reference plane for $(a)$ virtual origins $\lesssim 1$ wall unit and $(b)$ larger virtual origins. The velocity at the reference plane has a spanwise (horizontal arrow) and a wallnormal (vertical arrow) component.

Although not the focus of this paper, equations (2a)(2b) are also the result of a first order expansion when applying homogenisation on a given texture, and have been as such profusely studied (Philip, 1972; Lauga and Stone, 2003; Kamrin et al., 2010; Luchini, 2013). The expansion is typically done for the small parameter given by the ratio of the texture size to the flow thickness. In turbulent flows, it can be tempting to expand on the same parameter, but this does not produce satisfactory results. This is because the limiting parameter here is the ratio of the sizes of the texture and the near-wall eddies. Taking the quasi-streamwise vortices, they have diameters $\phi^{+} \approx 15$ and are centred at a height $y^{+} \approx 15$ above a smooth surface (Robinson, 1991; Schoppa and Hussain, 2002). In most applications of interest, this limiting parameter is then at least of order one, and smaller textures are typically considered hydraulically smooth (Jiménez, 2004). Higher order expansions are currently being investigated to circumvent this problem (Lācis et al., 2020; Bottaro, 2019; Bottaro and Naqvi, 2020).

The inherent assumption in the theory of Luchini (1996), given by equation (1), and the resulting slip boundary conditions (2), is that the quasi-streamwise vortices, which perceive a virtual smooth wall at $y^{+}=-\ell_{z}^{+}$, induce a spanwise velocity at the reference plane $y^{+}=0$, but zero wall-normal velocity. For $\ell_{z}^{+} \lesssim 1$, the wall-normal velocity induced at the reference plane would be negligible and the displacement of the vortices could indeed be effectively represented by the boundary condition ( $2 \mathrm{~b})$ plus zero $v$ transpiration, as sketched in figure $2(a)$. However, as $\ell_{z}^{+}$becomes comparable to the typical vortex diameter and vortexwall distance, which as mentioned in the previous paragraph are roughly 15 wall units, the wall-normal velocity that the vortices would induce at the reference plane would no longer be negligible, as sketched in fig-

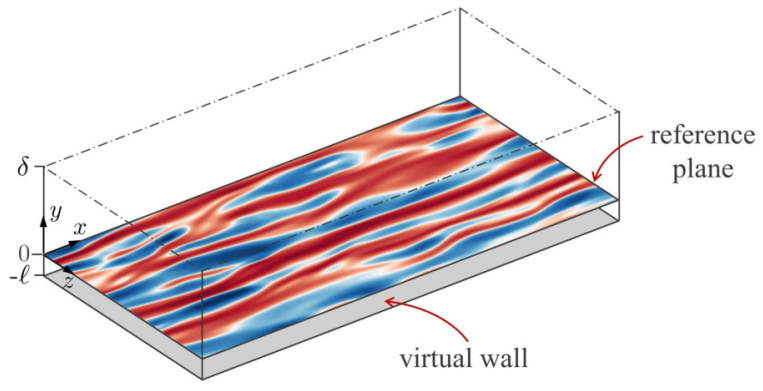

Figure 3: Channel flow with appropriate boundary conditions at the reference plane, $y=0$, so that the flow perceives the wall at the virtual wall, $y=-\ell$. The flow field at the reference plane corresponds to an instantaneous realisation of the streamwise velocity for case DHV444 in table 1 .

ure $2(b)$. Impeding $v$ at this plane would then restrict the displacement of the vortices. This effect can, for instance, be observed in the exhaustive set of slip-only simulations of Busse and Sandham (2012), for which Fairhall and García-Mayoral (2018) proposed an empirical expression to characterise the influence of impermeability. Gómez-de-Segura et al. (2018) suggested that some form of transpiration at $y^{+}=0$ would also need to exist for the quasi-streamwise vortices to perceive the origin at $y^{+}=-\ell_{z}^{+}$. When the condition on transpiration is effectively $v \approx 0$, as in the context of superhydrophobic surfaces (Min and Kim, 2004; Fukagata et al., 2006; Busse and Sandham, 2012), the origin the quasi-streamwise vortices would perceive would not be as deep as $y^{+}=-\ell_{z}^{+}$.

As mentioned before, in this work we explore the capability of different types of boundary conditions to impose virtual origins on DNSs of channel flows. The ultimate aim is to be able to set virtual origins for the three velocities independently, but as a first step we explore imposing the presence of a virtual smooth wall at $y^{+}=-\ell^{+}$, equal for all three velocities, as represented in figure 3. This will allow us to validate the models, as we can compare the flow with that in a smooth channel with the wall at $y^{+}=-\ell^{+}$. For instance, the mean velocity profile and all turbulence statistics should be smoothwall-like, except shifted in $y^{+}$by $\ell^{+}$. Once we identify a suitable technique, we will use it to impose different virtual origins for the different velocity components.

The paper is organised as follows. In section 2 we briefly describe the numerical method used. Section 3 presents and discusses the results from our simulations, exploring different techniques to impose virtual origins in section 3.1, and demonstrating the ability of the most suitable technique studied to impose different virtual 
origins on the three velocities in section 3.2. Conclusions are presented in section 4.

\section{Numerical method}

We conduct DNSs of symmetric channels with nonzero boundary conditions at both the bottom and top boundaries. The simulations conducted and their numerical parameters are summarised in tables 1 and 2 . The code used is adapted from Gómez-de-Segura and García-Mayoral (2019) and is only briefly summarised here. It solves the incompressible Navier-Stokes equations for the velocity $\boldsymbol{u}=(u, v, w)$ and the kinematic pressure $p$. The channels are periodic in $x$ and $z$, and their size is the standard $2 \pi \times \pi \times 2 \delta$ in the streamwise, spanwise and wall-normal directions, respectively, with the half-channel height $\delta=1$ measured from the reference plane $y=0$. This domain size has been shown to be adequate to capture one-point statistics up to the channel height for the friction Reynolds numbers used in the present study, as well as the flow dynamics that characterise the near-wall and log regions (LozanoDurán and Jiménez, 2014). The spatial discretisation is spectral in $x$ and $z$ and second-order centred finite differences in $y$. Simulations are conducted at constant pressure gradient, starting from a smooth-wall flow at friction Reynolds number $R e_{\tau}=u_{\tau} \delta / v \simeq 180$. The grid has $192 \times 192 \times 153$ collocation points with grid stretching in $y$, resulting in a resolution of $\Delta x^{+} \approx 5.9$, $\Delta z^{+} \approx 2.9$, and $\Delta y^{+} \approx 0.3$ near the wall stretching to $\Delta y^{+} \approx 3$ in the centre of the channel. The temporal integration uses the Runge-Kutta, fractional-step method of Le and Moin (1991). The boundary conditions are listed in table 1 and discussed in the next section. The coupling between velocity components, their wall-normal gradients and the pressure is fully implicit and embedded in the LU factorisation, intrinsic in the fractionalstep method (Perot, 1993). A detailed description of the implementation of this type of boundary conditions can be found in Gómez-de-Segura and García-Mayoral (2019) and Gómez-de-Segura (2019).

\section{Results and discussion}

Hereafter, we label the depth of the virtual origin perceived by the mean flow as $\ell_{U}^{+}$and that perceived by turbulence as $\ell_{T}^{+}$. These will be measured a posteriori in the DNS results. The depth $\ell_{U}^{+}$is given by the wall-normal displacement of the mean velocity profile with respect to a smooth-wall flow and, given that in viscous units $d U^{+} /\left.d y^{+}\right|_{y^{+}=0} \approx 1$, is essentially equal to the mean slip velocity, $\ell_{U}^{+} \approx U_{\text {slip }}^{+}$. The depth $\ell_{T}^{+}$, in contrast, will be estimated from the displacement of the Reynolds stress curve or that of the streamwise vorticity, $\omega_{x}^{\prime+}$. We will see later that these are essentially equal. Given that the local maximum in $\omega_{x}^{\prime+}$ near the wall is a good proxy for the location of the quasi-streamwise vortices (Choi et al., 1994), this supports the hypothesis of Luchini (1996) that the origin perceived by the vortices is also that perceived by turbulence.

\subsection{Modelling the presence of a smooth wall at $y^{+}=$ $-\ell^{+}$}

We begin by considering boundary conditions to model the presence of a smooth wall at $y^{+}=-\ell^{+}$. Such off-wall conditions have been considered, for instance, in Mizuno and Jiménez (2013) and Encinar et al. (2014) in the context of log-layer dynamics. Here we consider four cases, $\ell^{+}=1,2,3$ and 4 .

\subsubsection{Fully coupled boundary conditions from a Stokes-flow model}

For small $\ell^{+}$, such that the modelled region falls within the viscous sublayer, i.e. $\ell^{+} \leq 5$, the flow between the simulation boundary, $y^{+}=0$, and the virtual wall, $y^{+}=-\ell^{+}$, can be assumed to be dominantly viscous and can be modelled as a Stokes flow,

$$
-\nabla p+v \nabla^{2} \boldsymbol{u}=0 .
$$

We solve this equation analytically to yield the off-wall conditions at $y^{+}=0$ for the DNSs. For that, equation (3) is expanded using Fourier series in the wall-parallel directions, $x$ and $z$, and we consider the response of the modelled fluid region, $y^{+} \in\left[-\ell^{+}, 0\right]$, to the overlying interfacial shear and pressure waves in spectral space. This yields the following conditions at the reference plane $y^{+}=0$,

$$
\begin{aligned}
& \left.\hat{u}\right|_{y^{+}=0} ^{+}=\left.C_{u u}^{+} \frac{d \hat{u}}{d y}\right|_{y^{+}=0} ^{+}+\left.C_{u w}^{+} \frac{d \hat{w}}{d y}\right|_{y^{+}=0} ^{+}+\left.C_{u p}^{+} \hat{p}\right|_{y^{+}=0} ^{+}, \\
& \left.\hat{w}\right|_{y^{+}=0} ^{+}=\left.C_{w u}^{+} \frac{d \hat{u}}{d y}\right|_{y^{+}=0} ^{+}+\left.C_{w w}^{+} \frac{d \hat{w}}{d y}\right|_{y^{+}=0} ^{+}+\left.C_{w p}^{+} \hat{p}\right|_{y^{+}=0} ^{+}, \\
& \left.\hat{v}\right|_{y^{+}=0} ^{+}=\left.C_{v u}^{+} \frac{d \hat{u}}{d y}\right|_{y^{+}=0} ^{+}+\left.C_{v w}^{+} \frac{d \hat{w}}{d y}\right|_{y^{+}=0} ^{+}+\left.C_{v p}^{+} \hat{p}\right|_{y^{+}=0} ^{+},
\end{aligned}
$$

where the constants $C_{i j}^{+}$are complex and depend on the virtual origin $\ell^{+}$, as well as on the streamwise and spanwise wavelengths, $\lambda_{x}^{+}$and $\lambda_{z}^{+}$. The expressions for the upper wall (i.e. $y^{+}=2 \delta^{+}$) can be obtained by symmetry, and together they provide off-wall conditions for the 


\begin{tabular}{|c|c|c|c|c|}
\hline Case & $\left.\hat{u}^{+}\right|_{y^{+}=0}=$ & $\left.\hat{w}^{+}\right|_{y^{+}=0}=$ & $\left.\hat{v}^{+}\right|_{y^{+}=0}=$ & $R e_{\tau}$ \\
\hline FP222 & eq. (4a), $f\left(\alpha_{x}, \alpha_{z}\right)$ & eq. (4b), $f\left(\alpha_{x}, \alpha_{z}\right)$ & eq. (4c), $f\left(\alpha_{x}, \alpha_{z}\right)$ & 183 \\
\hline DIP222 & $C_{u u}^{+}\left(\alpha_{x}, \alpha_{z}\right) \frac{d \hat{u}^{+}}{d y}$ & $C_{w w}^{+}\left(\alpha_{x}, \alpha_{z}\right) \frac{d \hat{w}^{+}}{d y}$ & $C_{v p}^{+}\left(\alpha_{x}, \alpha_{z}\right) \hat{p}^{+}$ & 183 \\
\hline DHP222 & $\ell_{x}^{+} \frac{d \hat{u}^{+}}{d y}$ & $\ell_{z}^{+}{\frac{d \hat{w}^{+}}{d y}}^{+}$ & $C_{v p}^{+}\left(\alpha_{x}, \alpha_{z}\right) \hat{p}^{+}$ & 183 \\
\hline DHV222 & $\ell_{x}^{+} \frac{d \hat{u}^{+}}{d y}$ & $\ell_{z}^{+\frac{d \hat{w}}{d y}}+$ & $\ell_{y}^{+} \frac{d \hat{v}}{d y}^{+}$ & 182 \\
\hline DHV220 & $\ell_{x}^{+} \frac{d \hat{u}}{d y}{ }^{+}$ & $\ell_{z}^{+} \frac{d \hat{w}}{d y}{ }^{+}$ & 0 & 182 \\
\hline FP333 & eq. (4a), $f\left(\alpha_{x}, \alpha_{z}\right)$ & eq. (4b), $f\left(\alpha_{x}, \alpha_{z}\right)$ & eq. (4c), $f\left(\alpha_{x}, \alpha_{z}\right)$ & 184 \\
\hline DIP333 & $C_{u u}^{+}\left(\alpha_{x}, \alpha_{z}\right) \frac{d \hat{u}}{d y}^{+}$ & $\mathcal{C}_{w w}^{+}\left(\alpha_{x}, \alpha_{z}\right) \frac{d \hat{w}}{d y}^{+}$ & $C_{v p}^{+}\left(\alpha_{x}, \alpha_{z}\right) \hat{p}^{+}$ & 185 \\
\hline DHP333 & $\ell_{x}^{+\frac{d \hat{u}^{+}}{d y}}$ & $\ell_{z}^{+}{\frac{d \hat{w}^{+}}{d y}}^{+}$ & $C_{v p}^{+}\left(\alpha_{x}, \alpha_{z}\right) \hat{p}^{+}$ & 184 \\
\hline DHV333 & $\ell_{x}^{+\frac{d \hat{u}^{+}}{d y}}{ }^{+}$ & $\ell_{z}^{+} \frac{d \hat{w}^{+}}{d y}$ & $\ell_{y}^{+} \frac{d \hat{v}^{+}}{d y}$ & 183 \\
\hline DHV330 & $\ell_{x}^{+} \frac{d \hat{u}}{d y}{ }^{+}$ & $\ell_{z}^{+} \frac{d \hat{w}}{d y}{ }^{+}$ & 0 & 182 \\
\hline FP444 & eq. (4a), $f\left(\alpha_{x}, \alpha_{z}\right)$ & eq. (4b), $f\left(\alpha_{x}, \alpha_{z}\right)$ & eq. (4c), $f\left(\alpha_{x}, \alpha_{z}\right)$ & 186 \\
\hline DHP444 & $\ell_{x}^{+} \frac{d \hat{u}^{+}}{d y}$ & $\ell_{z}^{+} \frac{d \hat{w}^{+}}{d y}$ & $C_{v p}^{+}\left(\alpha_{x}, \alpha_{z}\right) \hat{p}^{+}$ & 185 \\
\hline DHV444 & $\ell_{x}^{+\frac{d \hat{u}^{+}}{d y}}{ }^{+}$ & $\ell_{z}^{+\frac{d y}{d y}}+$ & $\ell_{y}^{+} \frac{d \hat{v}^{+}}{d y}$ & 184 \\
\hline DHV440 & $\ell_{x}^{+} \frac{d \hat{u}}{d y}^{+}$ & $\ell_{z}^{+} \frac{d \hat{w}}{d y}{ }^{+}$ & 0 & 182 \\
\hline
\end{tabular}

Table 1: Boundary conditions for the three velocity components for each case simulated, where $R e_{\tau}$ is the friction Reynolds number measured either at the virtual wall, $y^{+}=-\ell^{+}$, when the three virtual origins are equal, or at the origin of turbulence, $y^{+}=-\ell_{T}^{+}$, otherwise. Each case is labelled with 2 or 3 letters followed by 3 digits. The letters refer to the equations used as boundary conditions and the numbers correspond to the desired values for the virtual origins for $u, w$ and $v$, respectively. For the first letter, $\mathrm{F}(u l l)$ means that the full Stokes conditions from equation (4) are used, and $\mathrm{D}$ (iagonal) means that only the three dominant terms, $C_{u u}, C_{w w}$ and $C_{v p}$ are used. The second letter denotes the type of boundary condition used for $u$ and $w$ : I(nhomogeneous) when wavelength-dependent coefficients from Stokes equation are considered, and $\mathrm{H}($ omogeneous $)$ when homogeneous slip lengths are considered. The last letter denotes the type of boundary condition used for $v$ : P when an impedance between $v$ and $p$ is imposed, derived from Stokes model, and $\mathrm{V}$ when $v=\ell_{y} d v / d y$ is used instead.

\begin{tabular}{lccccccc|ccc}
\hline \hline & $\ell_{u}^{+}$ & $\ell_{w}^{+}$ & $\ell_{v}^{+}$ & $\ell_{x}^{+}$ & $\ell_{z}^{+}$ & $\ell_{y}^{+}$ & $\ell_{s m}^{+}$ & $\ell_{U}^{+}$ & $\ell_{T}^{+}$ & $\ell_{T, \text { pred }}^{+}$ \\
\hline FP222 & 2.0 & 2.0 & 2.0 & - & - & - & - & 2.0 & 2.1 & - \\
DIP222 & 2.0 & 2.0 & 2.0 & - & - & - & - & 2.0 & 1.5 & - \\
DHP222 & 2.0 & $2.0(1.7)$ & 2.0 & 2.0 & 2.0 & - & - & 2.0 & 1.5 & - \\
DHV222 & 2.0 & $2.0(1.7)$ & 2.0 & 2.0 & 2.0 & 1.2 & 0.8 & 2.0 & 1.7 & 1.7 \\
DHV220 & 2.0 & $2.0(1.7)$ & 0.0 & 2.0 & 2.0 & 0.0 & 0.0 & 2.0 & 1.3 & 1.3 \\
\hline FP333 & 3.0 & 3.0 & 3.0 & - & - & - & - & 3.0 & $*$ & $*$ \\
DIP333 & 3.0 & 3.0 & 3.0 & - & - & - & - & 3.0 & $*$ & $*$ \\
DHP333 & 3.0 & $3.0(2.3)$ & 3.0 & 3.0 & 3.0 & - & - & 3.0 & $*$ & $*$ \\
DHV333 & 3.0 & $3.0(2.3)$ & 3.0 & 3.0 & 3.0 & 1.9 & 1.1 & 3.0 & 2.4 & 2.3 \\
DHV330 & 3.0 & $3.0(2.3)$ & 0.0 & 3.0 & 3.0 & 0.0 & 0.0 & 3.0 & 1.7 & 1.6 \\
\hline FP444 & 4.0 & 4.0 & 4.0 & - & - & - & - & 4.0 & $*$ & $*$ \\
DHP444 & 4.0 & $4.0(2.9)$ & 4.0 & 4.0 & 4.0 & - & - & 3.6 & $*$ & $*$ \\
DHV444 & 4.0 & $4.0(2.9)$ & 4.0 & 4.0 & 4.0 & 2.5 & 1.5 & 4.0 & 3.1 & 2.9 \\
DHV440 & 4.0 & $4.0(2.9)$ & 0.0 & 4.0 & 4.0 & 0.0 & 0.0 & 4.0 & 1.9 & 1.8 \\
\hline \hline
\end{tabular}

Table 2: Values of slip lengths and depths of virtual origins. Parameters specified a priori: the depths of virtual origins for the three velocities $\left(\ell_{u}^{+}, \ell_{w}^{+}\right.$and $\left.\ell_{v}^{+}\right)$, the slip lengths $\left(\ell_{x}^{+}, \ell_{z}^{+}\right.$and $\left.\ell_{y}^{+}\right)$, and $\ell_{s m}^{+}$, as defined in figure $9(b)$. For certain simulations we do not define any slip lengths, as a wavelength-dependent model is used instead. The values between parentheses for $\ell_{w}^{+}$correspond to the actual virtual origins that we impose when accounting for the curvature of $w^{\prime+}$. Parameters specified a posteriori: depth of the virtual origin for the mean flow, $\ell_{U}^{+}$, and that for turbulence, $\ell_{T}^{+}$, measured a posteriori from DNSs. $\ell_{T, \text { pred }}^{+}$corresponds to the origin of turbulence predicted using $\ell_{T, \text { pred }}^{+}=\ell_{w}^{+}$when $\ell_{w}^{+}<\ell_{v}^{+}$and equation (6) when $\ell_{w}^{+}>\ell_{v}^{+}$. The symbol '*' indicates that the simulation failed to model the effect of virtual origins, and we could not obtain them. 
flow within the channel. The dominant terms in equation (4) are those in the diagonal, that is $C_{u u}^{+}$and $C_{w w}^{+}$for $u^{+}$and $w^{+}$, which correspond to the slip lengths in the streamwise and spanwise directions, respectively, and $C_{v p}^{+}$for $v^{+}$, which is equivalent to the impedance coefficient of Jiménez et al. (2001) that relates the wallnormal velocity and the pressure.

To illustrate how the impedance for the wall-normal transpiration varies for different flow lengthscales, the transpiration coefficient $C_{v p}^{+}$for different wavelengths is portrayed in figure $4(a)$. Note that the Stokes equation is isotropic in the $x$ and $z$ directions, and therefore $C_{v p}^{+}$depends only on the spherical wavelength, $\hat{\lambda}=2 \pi / \sqrt{\alpha_{x}^{2}+\alpha_{z}^{2}}$, where $\alpha_{x}$ and $\alpha_{z}$ are the streamwise and spanwise wavenubers, respectively. This figure shows that the reference plane is essentially impermeable to large eddies, while the impermeability relaxes for smaller eddies and these can permeate through more easily. Additionally, figure 4(b) compares the transpiration coefficient, $C_{v p}^{+}$, and the spanwise slip coefficient, $C_{w w}^{+}$, experienced by eddies of the size of near-wall streaks (i.e. $\lambda_{x}^{+} \approx 1000$ and $\lambda_{z}^{+} \approx 100$ ) and of quasi-streamwise vortices (i.e. $\lambda_{x}^{+} \approx 100$ and $\lambda_{z}^{+} \approx 100$ ) for different $\ell^{+}$. It shows that both the streaks and quasi-streamwise vortices are subject to essentially the same slip length as the mean, $C_{w w}^{+}=\ell^{+}$. This, although not shown, is also the case for the streamwise slip coefficient $C_{u u}^{+}$. In contrast, the transpiration $C_{v p}^{+}$ is more sensitive to the wavelengths. Although $C_{v p}^{+}$is essentially zero for very large $\hat{\lambda}^{+}$, its value increases as the wavelengths decrease. This can be observed especially for $\ell^{+}=4$ in figure $4(b)$, where the surface is already significantly permeable for $\lambda_{z}^{+} \approx 100$. These observations will allow us to later simplify the off-wall conditions (4).

From the four virtual origins under study, $\ell^{+}=1,2,3$ and 4 , the first two exhibit proportionately the same behaviour. Therefore, for clarity only results for $\ell^{+}=2,3$ and 4 are presented. These are labelled as cases FP222, FP333 and FP444, respectively, where the digits correspond to the sought values for the virtual origins for $u$, $w$ and $v$, respectively, and the letters refer to the model used. The first letter F(ull) means that the full Stokes conditions from equation (4) are used, and the second letter, $\mathrm{P}($ ressure $)$, means that the boundary condition for $v$ uses a fully implicit coupling between $v$ and $p$.

To assess if these cases successfully model a smooth wall at $y^{+}=-\ell^{+}$, figure 5 portrays one-point statistics obtained from the DNSs. In the panels on the left, panels (a.1-a.4), the mean velocity profile and the turbulent fluctuations are represented in the conventional form, with the origin of the wall-normal height at the reference plane, $y^{+}=0$, and scaled with $u_{\tau}$ measured at that height. In this framework, in panel (a.1) the non-zero slip velocity, $U_{\text {slip }}^{+}$, is observed at the reference plane, $y^{+}=0$, which is essentially the depth of the virtual origin for the mean flow, $\ell_{U}^{+}$. This shift, however, does not extend along the entire $y$-range. The shift of the mean profile in the logarithmic region is significantly smaller than $U_{\text {slip }}^{+}$and for cases FP333 and FP444 it even becomes negative, revealing the adverse effect of the boundary conditions on $\Delta U^{+}$and hence the drag. Additionally, the rms velocity profiles, the Reynolds stresses and the streamwise vorticity in panels (a.2-a.4) are displaced towards the reference plane and show a change in magnitude compared to a smooth channel.

Since the aim was to model a smooth wall at $y^{+}=$ $-\ell^{+}$, smooth-channel profiles with an origin at $y^{+}=-\ell^{+}$ should be recovered. This origin implies that $u_{\tau}$ should be obtained from the total stress at that height. This cannot be measured directly, but the total stress profile within the channel is linear and it can be extrapolated to $y=-\ell$. The friction velocity at that height is then $u_{\tau}=u_{\tau_{y=0}}(1+\ell / \delta)^{1 / 2}$. The effective half-channel height, in turn, becomes $\delta^{\prime}=\delta+\ell$. Defined this way, the only difference between the profiles should be the offset in $y^{+}$by their origin, $\ell^{+}$, and shifting them should give a collapse of all the turbulence profiles with the smooth-wall data. This is how results are represented in panels (b.1-b.4). At a first glance, the profiles suggest that the boundary conditions of equation (4) provide a good model for $\ell^{+} \lesssim 2$, as all the curves for case FP222 in panels (b.1-b.4) show an excellent collapse with smooth-wall data. Note that in the panels on the left, the peaks of the fluctuations for case FP222 show a slight increase in magnitude with respect to a smooth wall. This is an artefact of the scaling, and all deviations from the smooth-wall data vanish when values are scaled by the friction velocity measured at $y^{+}=-2$. This highlights the relevance of the scaling for the interpretation of the data. For larger $\ell^{+}$, however, the Stokes model fails to mimic a smooth wall at $y^{+}=-\ell^{+}$, as shown by the deviations of the mean velocity profile and the turbulent intensities for cases FP333 and FP444 in panels 5(b.1-b.4). For these cases, the model does not merely shift the overlying flow by the virtual origin, but it also modifies it.

To gain further insight on whether the intensity of the one-point statistics arises from a mere change in intensity or a redistribution of energy, we examine the spectral density distributions of $u^{2}, v^{2}$ and $w^{2}$. As an example, figure 6 shows the energy distribution at a 

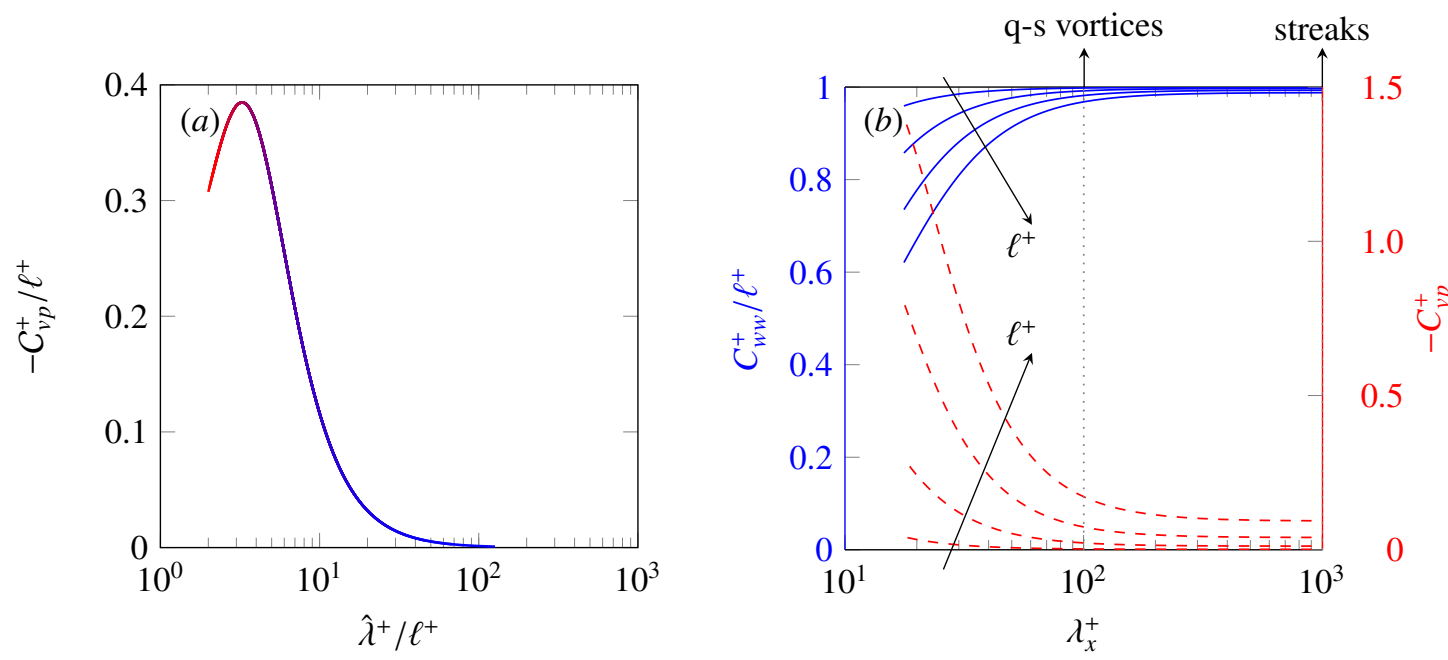

Figure 4: (a) Dimensionless transpiration coefficient $C_{v p}$ as a function of the spherical wavelength $\hat{\lambda}=2 \pi / \sqrt{\alpha_{x}^{2}+\alpha_{z}^{2}}$, both scaled with the depth of the virtual wall, $\ell^{+}$. The curve is a collapse of several curves with different values of $\ell^{+}$, from blue to red $\ell^{+} \in[0.1-4]$. (b) --- , transpiration coefficient $C_{v p}^{+} ;$, spanwise slip coefficient $C_{w w}^{+} / \ell^{+}$, for $\lambda_{z}^{+} \approx 100$ as a function of $\lambda_{x}^{+}$for different virtual origins $\ell^{+}=1,2,3$ and 4 . Arrows indicate increasing $\ell^{+}$and the vertical dotted lines correspond to lengthscales of the size of streaks and quasi-streamwise vortices.

height of roughly 10 wall units above the virtual origin, $y^{+}+\ell^{+} \approx 10$. For $\ell^{+}=2$, the agreement with the smooth channel is excellent, confirming that turbulence remains essentially smooth-wall-like and that the effect of the boundary conditions (4) is to ascribe the position of a smooth wall to $y^{+}=-\ell^{+}$. For cases FP333 and FP444, however, there are some differences. Panels $(d-i)$ reveal that the variations in intensity in the onepoint statistics are caused by contributions from lengthscales that are not significant over smooth walls. The wall-normal velocity exhibits the most dramatic change. Panels $(e)$ and $(h)$ show the appearance of a new energetic region, short in $x$ and wide in $z$. These lengthscales are also appreciable in the instantaneous realisations of $u^{+}$and $v^{+}$portrayed in figure 7 . We observe that, although the velocity fields for case FP222 in panels $(c-d)$ resemble those over a smooth wall, where the $u$-field displays the signature of the streaks and the $v$-field that of quasi-streamwise vortices, the fields for case FP444 in panels $(e-f)$ are very different and show the appearance of new lengthscales. This can be attributed to the large values of the impedance coefficient $C_{v p}^{+}$assigned to small lengthscales, as observed in figure $4(a)$, which excites short modes. This will be further analysed in the following subsections. Notice also that for cases FP333 and FP444, it is not possible to obtain a virtual origin for turbulence per se, as by definition the concept of a virtual origin assumes that the near-wall cycle and the turbulence dynamics remain smooth-wall-like, which is no longer the case. This is why table 2 does not provide a value for $\ell_{T}^{+}$for cases FP333 and FP444.

\subsubsection{Uncoupled boundary conditions from a Stokes- flow model}

The implementation of the boundary conditions from equation (4) involves the full coupling between the three velocities and the pressure, which would make setting different virtual origins for each velocity component difficult. In what follows, we neglect certain terms from equation (4), while still maintaining a common virtual origin at $y^{+}=-\ell^{+}$. The aim is to study the decoupling of the boundary conditions on the three velocity components to later impose different virtual origins. We do so by retaining only the dominant terms for each component, that is, the slip coefficients $C_{u u}^{+}$and $C_{w w}^{+}$for $u^{+}$and $w^{+}$, respectively, and the transpiration coefficient $C_{v p}^{+}$ for $v^{+}$. Thus, the boundary condition for each velocity component is independent of the other two components. For this model, only virtual origins with $\ell^{+}=2$ and 3 were tested, labelled as DIP222 and DIP333, where $\mathrm{D}$ (iagonal) denotes that only the three coefficients in the diagonal of equation (4) are retained, I(nhomogeneous) denotes that the coefficients for $u$ and $w$ are wavelengthdependent, and $\mathrm{P}$ denotes that the boundary condition for $v$ is imposed in terms of $p$. As before, the digits correspond to the virtual origins for $u, w$ and $v$ that we intend to impose.

For $\ell^{+}=2$, case DIP222, the model reproduces reasonably well the effect of a virtual wall at $y^{+}=-2$, as 

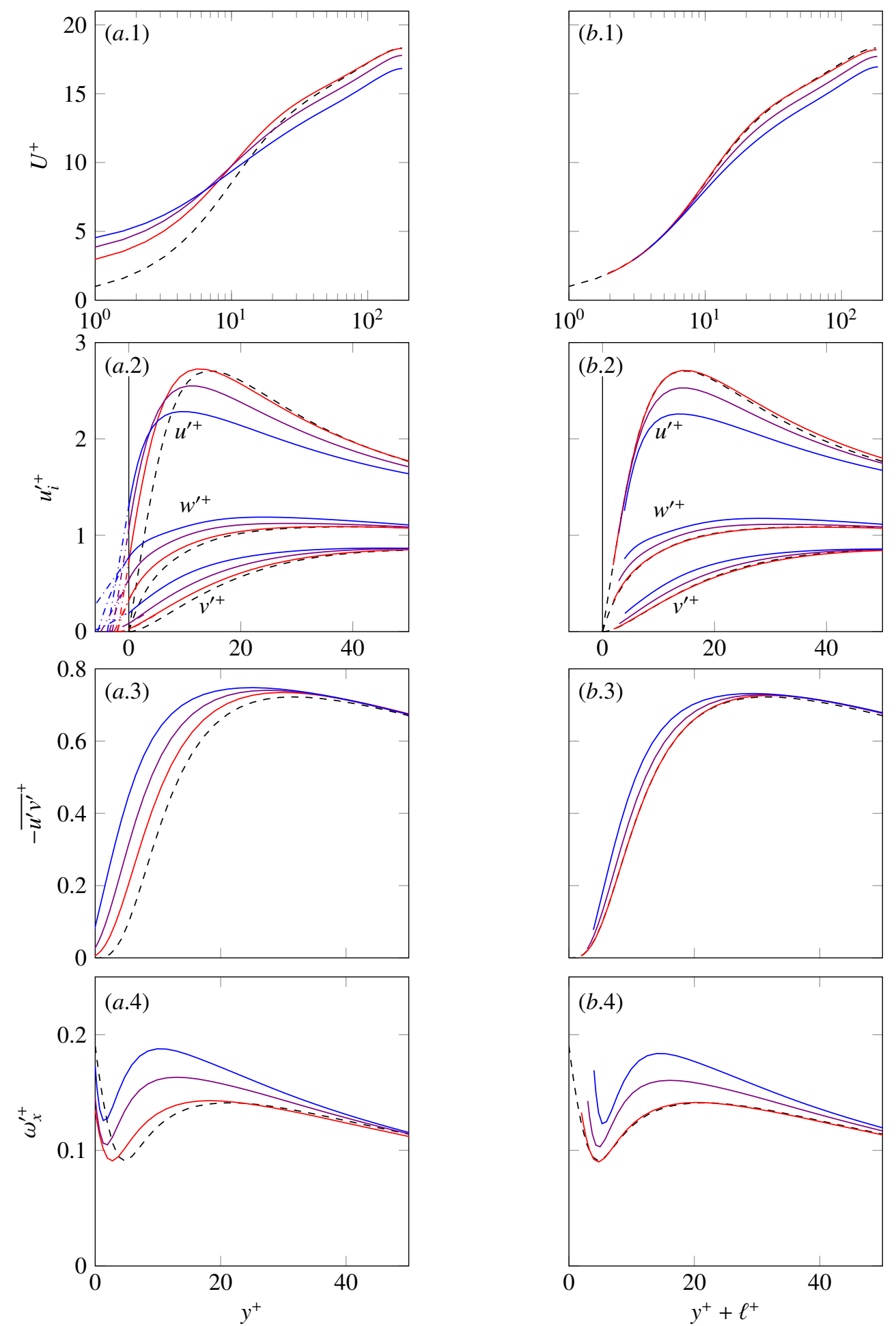

Figure 5: First column (a.1-a.4), profiles scaled with $u_{\tau}$ measured at the reference plane where the boundary conditions are imposed, $y^{+}=0$. Second column (b.1-b.4), profiles scaled with $u_{\tau}$ measured at the virtual wall, $y^{+}=-\ell^{+}$, and shifted by $\ell^{+}$. - - - -, smooth channel; FP333; —_ FP444. (a.1,b.1) Mean velocity profiles; $(a .2, b .2)$ rms velocity fluctuations; $(a .3, b .3)$ Reynolds shear stress; (a.4,b.4) streamwise vorticity fluctuations. 

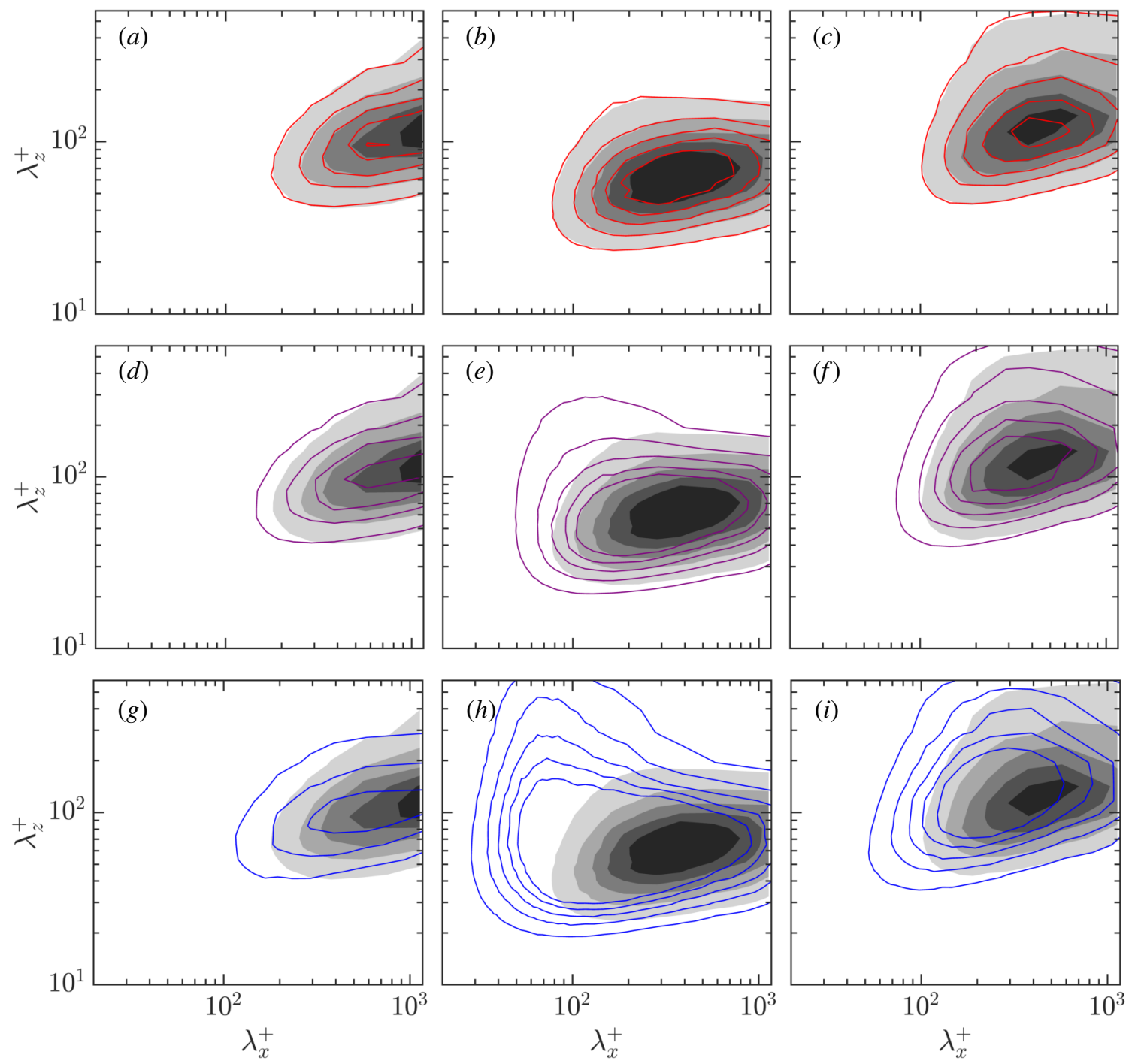

Figure 6: Premultiplied two-dimensional spectral densities of $u^{2}, v^{2}$ and $w^{2}$, referred to as $k_{x} k_{z} E_{u u}, k_{x} k_{z} E_{v v}$ and $k_{x} k_{z} E_{w w}$, respectively. Shaded contours correspond to the smooth channel at $y^{+} \approx 10$ and the solid lines to the DNSs shifted by their corresponding virtual origin, i.e. at $y^{+} \approx 10-\ell^{+}$, and scaled with $u_{\tau}$ measured at $y^{+}=-\ell^{+}$. $(a-c) \longrightarrow$, FP222; $(d-f) \longrightarrow$, FP333; $(g-i) \longrightarrow$, FP444. First column, $k_{x} k_{z} E_{u u}$; second column $k_{x} k_{z} E_{v v}$; third column, $k_{x} k_{z} E_{w w}$. The contour increments in wall units are $0.3241,0.0092$ and 0.0404 , respectively.

shown by the good collapse of the profiles with smoothwall data illustrated in figures 8(a.1-a.4). On a closer inspection, however, the turbulent fluctuations show a small, but noticeable, deviation from the smooth-wall data. In particular, the depth of the virtual origin of $w$ and $v$ is slightly less than 2 wall-units and, as a result, the shift of the streamwise vorticity and the Reynolds stress, which relate to the origin for quasi-streamwise vortices, is also slightly less than 2 wall units, approximately, $\ell_{T}^{+} \approx 1.5$ (see table 2 ). The origin for the mean flow, on the other hand, is at $y^{+}=-2$. The difference between the virtual origin perceived by the mean flow and that perceived by the quasi-streamwise vortices results in a small upward shift of the log law, as can be appreciated in panel (a.1).

For $\ell^{+}=3$, on the other hand, results are, as expected, no better than those previously observed with full offwall conditions, case FP333. The current model also fails to mimic a virtual origin at $y^{+}=-3$. 
(a)

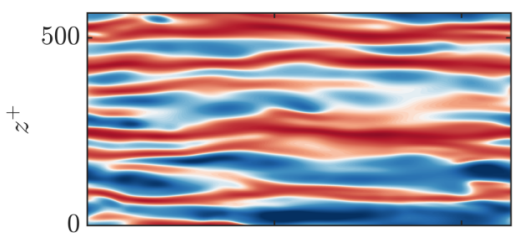

(b)

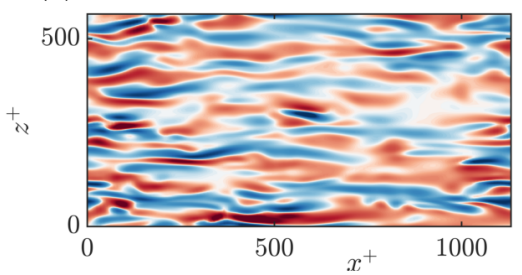

(c)

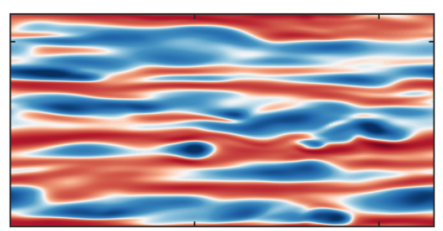

(d)

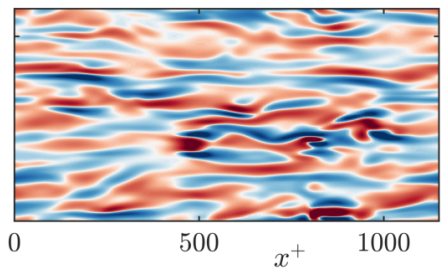

(e)

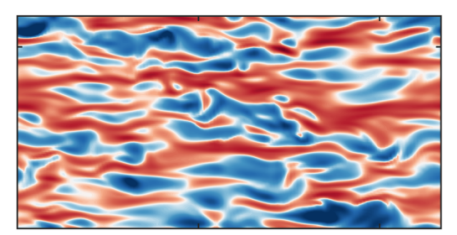

(f)

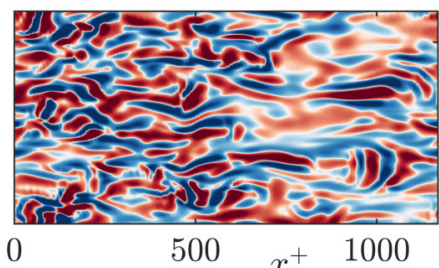

Figure 7: Instantaneous realisations of streamwise, $u^{\prime+}$, (top) and wall-normal, $v^{++}$, (bottom) velocities at a plane parallel to the wall. ( $a$ - $b$ ) Smooth channel at $y^{+} \approx 7$. $(c-d)$ Case FP222 at $y^{+} \approx 7-\ell^{+} \approx 5$. $(e-f)$ Case FP444 at $y^{+} \approx 7-\ell^{+} \approx 3$. In all cases, red to blue corresponds to [-5.0, +5.0] for $u^{\prime+}$ and $[-0.48,+0.48]$ for $v^{\prime+}$.

\subsubsection{Uncoupled boundary conditions from a Stokes- flow model with homogeneous slip lengths}

To further simplify the boundary conditions, we now impose homogeneous tangential slip lengths $\left(\ell_{x}^{+}\right.$and $\left.\ell_{z}^{+}\right)$to model the virtual origins of $u$ and $w$, removing any dependencies of the slip coefficients on the lengthscales. For $v$, on the other hand, we keep a wavelengthdependent transpiration, $C_{v p}^{+}$, as before. This follows from previous studies on slip-only simulations (Min and Kim, 2004; Busse and Sandham, 2012), and is also supported by the observations in the preceding discussion regarding figure $4(b)$. This figure shows how $C_{w w}^{+}$is essentially homogeneous across the energetically relevant lengthscales in the flow, and assuming it to be homogeneous is therefore a sensible approximation. The same can also be argued for $C_{u u}^{+}$, but not $C_{v p}^{+}$. For the latter, its wavelength-dependent nature is significant in the characteristic range of near wall turbulence and would thus need to be accounted for. We refer to these simulations as DHP222, DHP333 and DHP444 where, compared to the previous cases, the second letter has been switched to $\mathrm{H}$ (omogeneous) to denote that the boundary conditions for $u$ and $w$ are now homogeneous, that is, the same for all wavelengths.

The results obtained with this model are similar to those in the previous subsection, as shown in figures $8(b .1-b .4)$. For $\ell^{+}=2$, case DHP222 mimics reasonably well a wall at $y^{+}=-2$, although, as for DIP222, the origin perceived by quasi-streamwise vortices is slightly shallower than 2 wall units. For larger $\ell^{+}$, cases DHP333 and DHP444 also failed to model a virtual wall at $y^{+}=-3$ and $y^{+}=-4$, respectively. Additionally, with uncoupled boundary conditions, the pro- files of $v^{\prime+}$ near the wall diverge even more than when full Stokes-flow conditions were imposed, i.e. in cases FP333 and FP444 from before. Although not shown here, with uncoupled boundary conditions the new energetic region observed previously in the $v$-spectrum of FP333 and FP444 intensifies, supporting the idea that the disparity between these cases and the corresponding smooth channel is indeed due to the large permeability values at small lengthscales. In order to eliminate the appearance of the new lengthscales observed for DHP333 and DHP444, a low-pass filtering on $C_{v p}^{+}$ was also tested in a separate simulation. In this case, the boundary condition $v^{+}=C_{v p}^{+} p^{+}$was applied only to larger eddies, while eddies smaller than $\lambda_{x}^{+}<50$ and $\lambda_{z}^{+}<10$ were free to permeate through a boundary condition of the form $d v^{+} / d y^{+}=0$, avoiding possible feedback amplification between $v$ and $p$. However, this was not sufficient to impede the amplification of all the small lengthscales.

\subsubsection{Robin conditions for the three velocities}

Given that the model constructed from the Stokes equation breaks down for a virtual origin deeper than two wall units, which would be insufficient to model a sufficiently wide range of virtual origins, a different strategy was investigated. By analogy with the sliplength model for the tangential velocities, we propose 

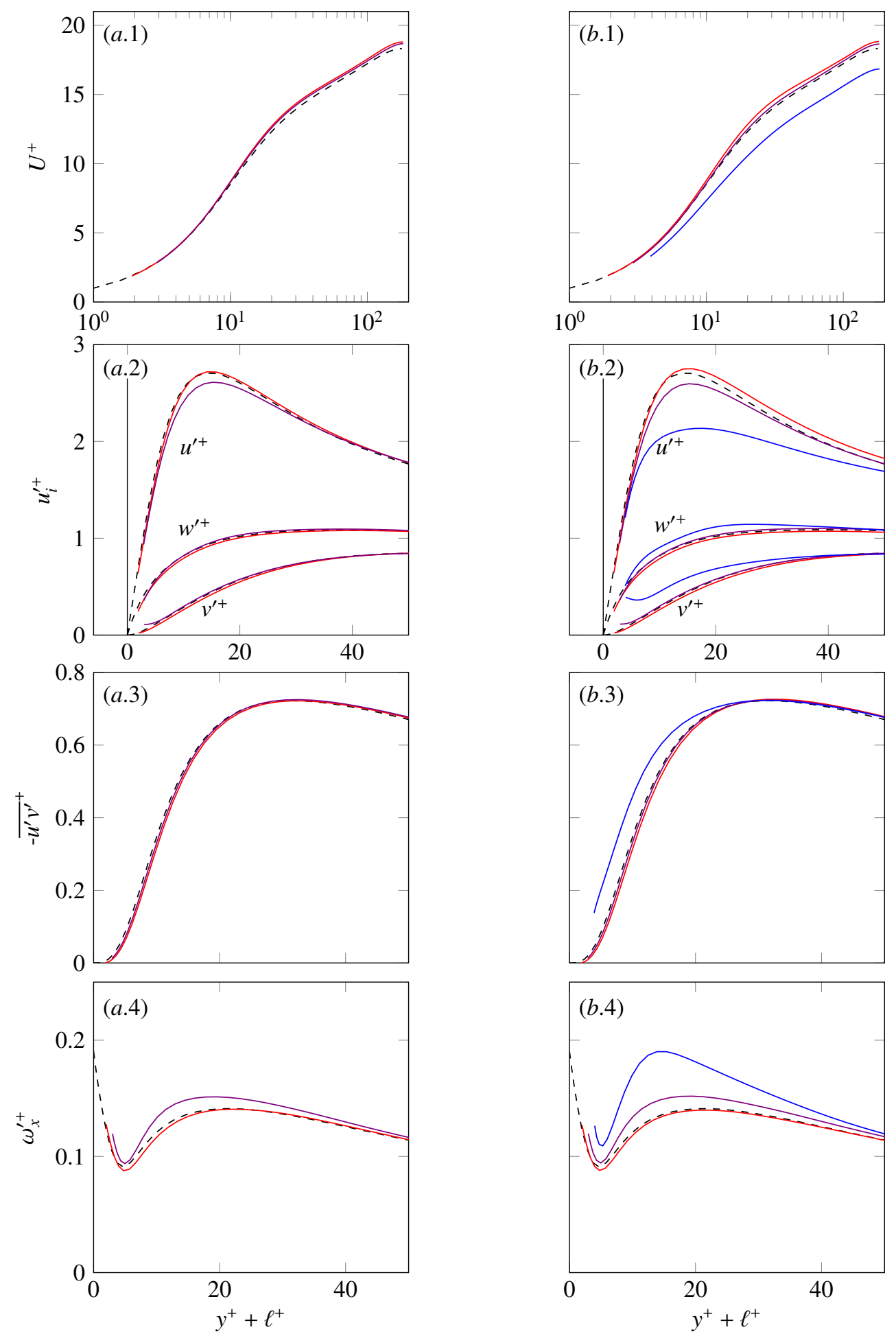

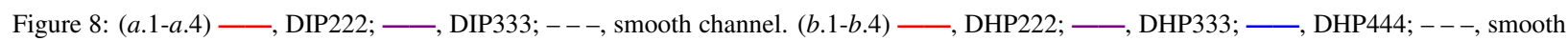
channel. (a.1-b.1) Mean velocity profiles; (a.2-b.2) rms velocity fluctuations; (a.3-b.3) Reynolds shear stresses; (a.4-b.4) streamwise vorticity fluctuations. All the profiles are scaled with $u_{\tau}$ measured at the virtual wall, $y^{+}=-\ell^{+}$, and shifted by $\ell^{+}$. 
Robin conditions for the three velocity components,

$$
\begin{gathered}
\left.u\right|_{y=0}=\left.\ell_{x} \frac{\partial u}{\partial y}\right|_{y=0}, \\
\left.w\right|_{y=0}=\left.\ell_{z} \frac{\partial w}{\partial y}\right|_{y=0}, \\
\left.v\right|_{y=0}=\left.\ell_{y} \frac{\partial v}{\partial y}\right|_{y=0},
\end{gathered}
$$

so that $u, w$ and $v$ are related to their respective wallnormal gradients by three slip lengths, $\ell_{x}, \ell_{z}$ and $\ell_{y}$. Note that for $v, \ell_{y}$ does not convey a slip effect, but, by extension, in the present work, we also refer to $\ell_{y}$ as the 'slip length' in the wall-normal direction. In what follows, we will set a homogeneous $\ell_{y}^{+}$, that is, equal for all wavelengths, except for the mean (i.e. $\lambda_{x} \rightarrow \infty$ and $\lambda_{z} \rightarrow \infty$ ), which from continuity must be zero.

While the concepts of slip lengths and virtual origins have been used interchangeably in the literature, here we make a subtle, but important, difference. We will denote by $\ell_{x}^{+}, \ell_{z}^{+}$and $\ell_{y}^{+}$the slip lengths in the streamwise, spanwise and wall-normal directions, respectively, defined as the proportionality coefficients in Robin boundary conditions (5). From a physical point of view, the slip lengths simply correspond to the $y$-locations where the velocity components become zero when linearly extrapolated from the reference plane. In contrast, we define the virtual origins of $u, w$ and $v$ as the locations below the reference plane where each velocity component perceives a virtual, smooth wall. These are at $y^{+}=-\ell_{u}^{+}$, $y^{+}=-\ell_{w}^{+}$and $y^{+}=-\ell_{v}^{+}$, respectively. These correspond to the heights where the rms profiles of the velocity fluctuations $u^{\prime+}, w^{\prime+}$ and $v^{\prime+}$ would go to zero assuming that the shape of the profiles remained smoothwall-like independently from each other, as illustrated in figure $9(a)$. The slip lengths for the Robin boundary conditions are therefore chosen according to the virtual origins that we intend to impose, $\ell_{u}^{+}, \ell_{w}^{+}$and $\ell_{v}^{+}$, which in the framework of modelling the presence of a smooth wall would be $\ell_{u}^{+}=\ell_{w}^{+}=\ell_{v}^{+}=\ell^{+}$. For a virtual origin of a few wall units, we expect the slip lengths $\ell_{x}^{+}$and $\ell_{z}^{+}$ to be approximately equal to the origins $\ell_{u}^{+}$and $\ell_{w}^{+}$, as the tangential velocities $u^{+}$and $w^{+}$are essentially linear in the immediate vicinity of the wall. We therefore assume that $\ell_{x}^{+} \approx \ell_{u}^{+}$and $\ell_{z}^{+} \approx \ell_{w}^{+}$, and set $\ell_{x}^{+}$and $\ell_{z}^{+}$equal to 2, 3 and 4 . The case of $v$, however, is slightly more delicate. Due to $v^{\prime}$ being quadratic with the distance to the wall, the depth of the virtual origin of $v, \ell_{v}^{+}$, differs from the slip length $\ell_{y}^{+}$, even for small values. We set $\ell_{y}^{+}$by matching the ratio between $v^{\prime+}$ and $\mathrm{d} v^{+} / \mathrm{d} y^{+}$ at the boundary plane with that at a height $\ell_{v}^{+}$above a smooth wall. From figure $9(b)$, we can observe that $\ell_{y}^{+}$ and $\ell_{v}^{+}$relate to each other by $\ell_{y}^{+}=\ell_{v}^{+}-\ell_{s m}^{+}$, where $\ell_{s m}^{+}$ is obtained from extrapolating the gradient of $v^{\prime+}$-profile from a smooth-wall DNS at $y^{+}=\ell_{v}^{+}$. This is the depth where, from the point of view of the plane $y^{+}=\ell_{v}^{+}$, the $v^{\prime+}$-profile would extrapolate to zero. Note that the value of $\ell_{s m}^{+}$is a function of $\ell_{v}^{+}$, as it depends on the local gradient at the height at which the extrapolation of the profile is calculated. The values of the slip lengths and virtual origins for the simulations here analysed are compiled in table 2. These cases are labelled DHV222, DHV333 and DHV444, where the third letter has been switched to $\mathrm{V}$ to indicate that the boundary condition for $v$ is now set by a Robin boundary condition in terms of $v$ alone.

Comparing to the models investigated in the previous sections, this technique produces a significant improvement on the results, as shown by the good collapse of the turbulent profiles in figure 10 and the spectral energy distributions in figure 11, as well as by the similarity in the instantaneous flow fields between the three cases illustrated in figure 12 . This model reproduces with reasonable accuracy not only a virtual origin at $y^{+}=-2$, but also at $y^{+}=-3$ and $y^{+}=-4$. However, on a closer inspection, the resulting origins for the transverse velocities, and consequently the origin for turbulence, $y^{+}=-\ell_{T}^{+}$, are slightly shallower than expected (see table 2). This is due to the curvature of $w^{+}$, which was neglected when assuming $\ell_{w}^{+}=\ell_{z}^{+}$. Beyond $y^{+} \gtrsim 1, w^{+}$is not exactly linear, and hence the origin of the spanwise velocity actually imposed is shallower than originally intended, as can be appreciated in figure 13. This suggests that we should have accounted for the curvature of $w^{\prime}$, and defined $\ell_{z}^{+}$in a similar fashion to $\ell_{y}^{+}$. In retrospect, contemplating the effect of the curvature, the depth of the origin of the spanwise velocity for cases DHV222, DHV333 and DHV444 is $\ell_{w}^{+}=1.7$, 2.3 and 2.9 , respectively, as indicated by the values between parentheses in table 2 . This effect of the curvature, although weaker, is also present for ${u^{\prime}}^{+}$, but for the origins here considered, $\ell^{+} \lesssim 4$, this effect is negligible. Considering $\ell_{x}^{+}=\ell_{u}^{+}$remains therefore an accurate approximation.

In these simulations, the displacement of quasistreamwise vortices towards the reference plane would be limited by the spanwise velocity, since $\ell_{w}^{+}$is the most restrictive origin between those of $v$ and $w$, i.e. $\ell_{w}^{+}<\ell_{v}^{+}$. This is evidenced from the displacement of the streamwise vorticity and Reynolds stress curves, where the origin of turbulence is found to be approximately coincident with that of $w, \ell_{T}^{+} \approx \ell_{w}^{+}$. Therefore, when $\ell_{w}^{+}<\ell_{v}^{+}$, the origin for turbulence could be predicted by $\ell_{w}^{+}$, i.e. $\ell_{T, \text { pred }}^{+}=\ell_{w}^{+}$, as can be observed in table 2 . 

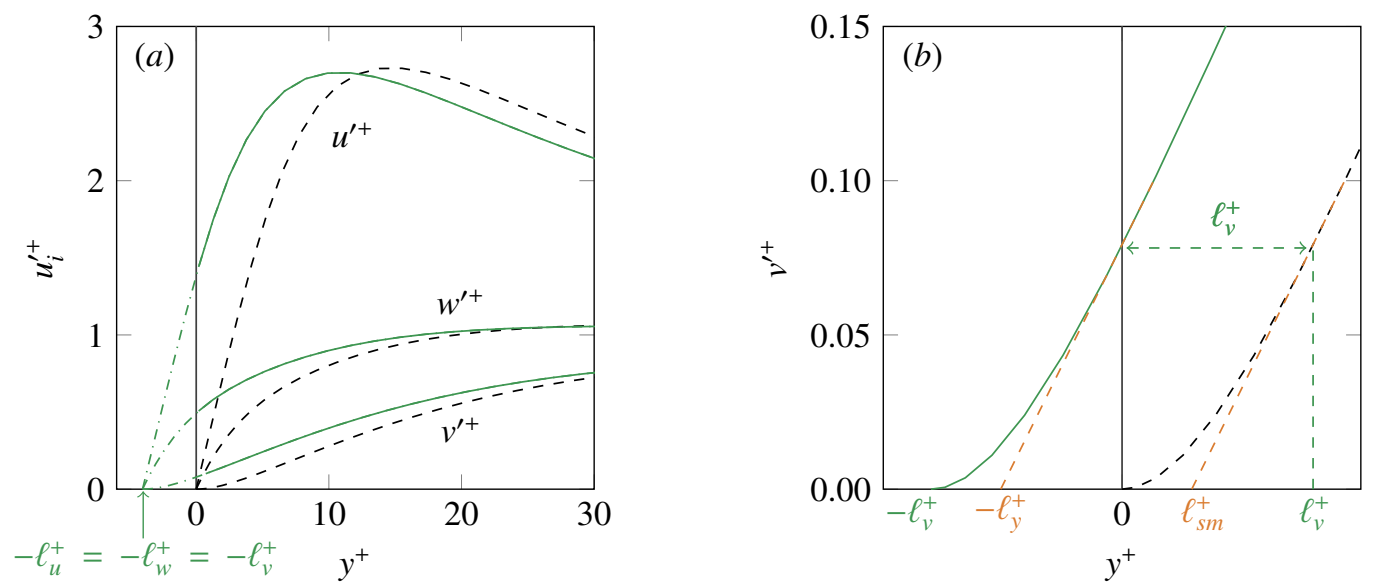

Figure 9: Schematics showing (a) the definition of the depths of the virtual origins $\ell_{u}^{+}, \ell_{w}^{+}$and $\ell_{v}^{+}$as the shift of the rms velocity fluctuations with respect to a smooth channel; $(b)$ the difference between $\ell_{v}^{+}$and $\ell_{y}^{+}$. - , smooth channel; - , curves with equal origins for the three velocity components.

Moreover, when displaced by $\ell_{T}^{+}$, the one-point statistics in figure 10 and the spectral density distributions of $u^{2}, v^{2}$ and $w^{2}$ in figure 11 show a better collapse with the smooth-wall data than when displaced by their corresponding virtual origins. This suggests that the nearwall cycle, typically at heights $y^{+}+\ell_{T}^{+} \approx 10-25$, is displaced in block by the origin perceived by turbulence, $\ell_{T}^{+}$, even though at the reference plane the velocity components decay to their respective virtual origins, as enforced by the Robin boundary conditions. Thus, in figures 10 and 11 we use $y^{+}=-\ell_{T}^{+}$as the origin for the wall-normal height, with $u_{\tau}$ measured at that origin.

While the turbulent fluctuations perceive an origin at $y^{+}=-\ell_{T}^{+}$, the origin for the mean flow agrees with the streamwise slip length, i.e. $\ell_{U}^{+}=\ell_{x}^{+}$, as the mean velocity profile is much closer to linear in the viscous sublayer than $w^{\prime+}$ or $v^{\prime+}$. It follows from the mean streamwise momentum equation for $U(y)$ that the mean velocity profile is determined by the overlying turbulence through the Reynolds shear stress (García-Mayoral and Jiménez, 2011; García-Mayoral et al., 2019). If the only effect of the virtual origins on turbulence, and hence on the Reynolds stress, is shifting its origin to $y^{+}=-\ell_{T}^{+}$, the only change in the mean velocity profile $U^{+}$compared to a smooth wall would be a shift by its value at $y^{+}=-\ell_{T}^{+}$. Above this height, the $U^{+}$-profile would curve up as it does over a smooth wall, while below it would remain essentially linear with $y^{+}$. The slight upward shift of the logarithmic region compared to a smooth wall can then be attributed to the difference in the origin perceived by the mean flow and by turbulence, $\ell_{U}^{+}-\ell_{T}^{+}$, and subtracting this difference gives a col- lapse of the velocity profiles, as shown in figure 10(b.1). This confirms the original idea from Luchini (1996) that "the law of the wall describing the mean-flow profile of the turbulent stream is modified by the presence of riblets only through a displacement of the origin of an amount equal to the protrusion-height difference. This is what one could expect if the structure of turbulent eddies were unaltered in the reference frame that has the transverse equivalent wall as origin, whereas the mean flow profile obviously starts at the longitudinal equivalent wall," with the small correction that the origin perceived by turbulence is not directly at $y^{+}=-\ell_{z}^{+}$.

The differences between the virtual origins of the three velocity components and turbulence in the cases studied, however, are too narrow for the evidence to be conclusive. The hypothesis that turbulence is displaced in block by $\ell_{T}^{+}$needs to be further tested by imposing significantly different virtual origins for the different velocity components. This is preliminarily investigated in the next section.

\subsection{Different virtual origins for the tangential and wall-normal velocities with Robin boundary con- ditions}

Now that we have found a suitable technique to impose a virtual origin for the three velocities, we can investigate setting different origins for each component and how they affect the overlying flow. In this section, we consider cases with $\ell_{x}^{+}=\ell_{z}^{+}=2,3$ and 4 as before, but with $v=0$ at the reference plane $y=0$, i.e. conventional slip-only simulations as those of Busse and Sandham (2012) and Min and Kim (2004). These cases 

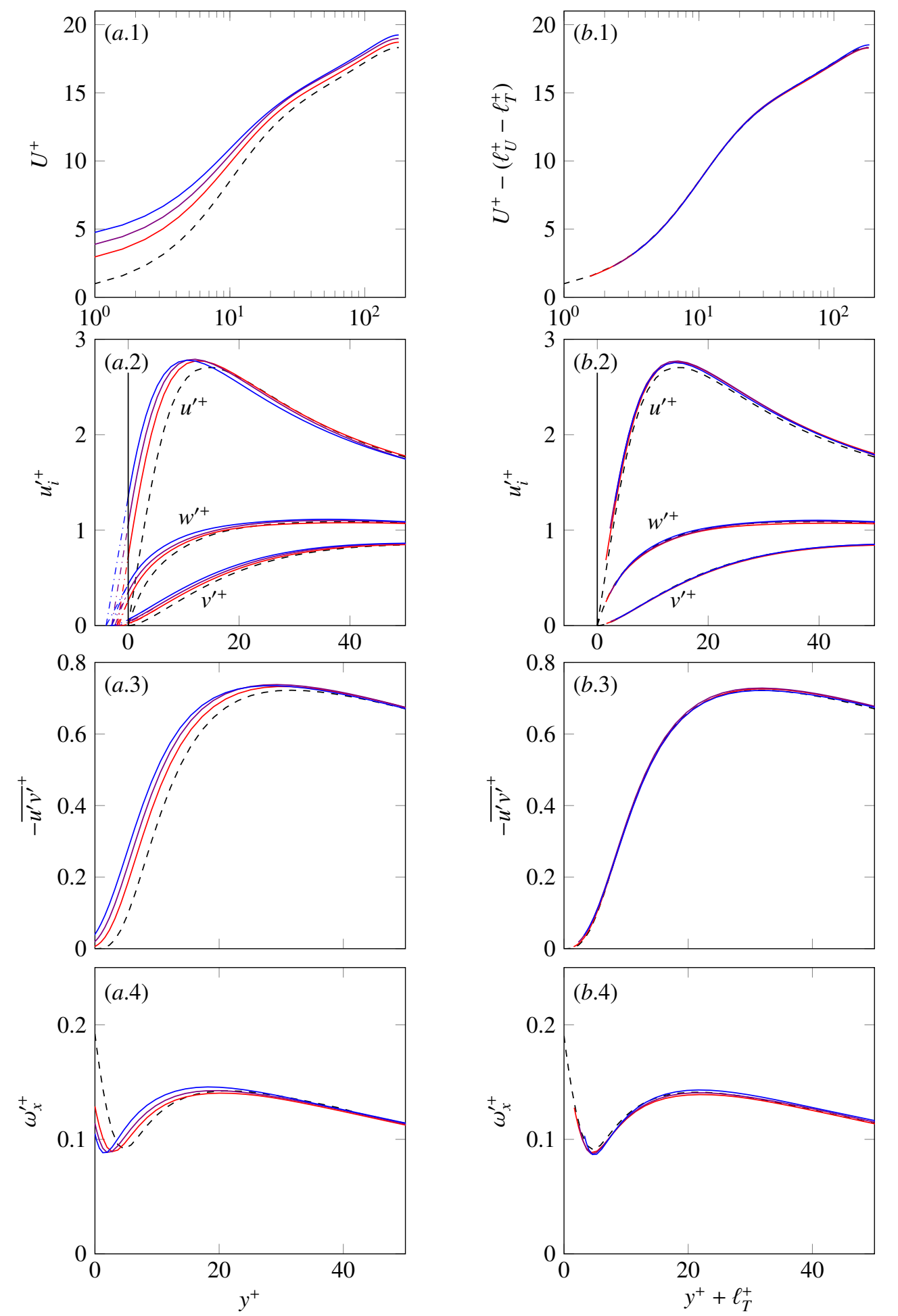

Figure 10: First column (a.1-a.4), profiles scaled with $u_{\tau}$ measured at the reference plane where the boundary conditions are imposed, $y^{+}=0$. Second column (b.1-b.4), profiles scaled with $u_{\tau}$ measured at the origin of turbulence, $y^{+}=-\ell_{T}^{+}$, and shifted by $\ell_{T}^{+}$. -- - , smooth channel; - , DHV222; — D DHV333; —_ DHV444. ( $a .1, b .1)$ Mean velocity profiles; $(a .2, b .2)$ rms velocity fluctuations; (a.3,b.3) Reynolds shear stresses; $(a .4, b .4)$ streamwise vorticity fluctuations. 

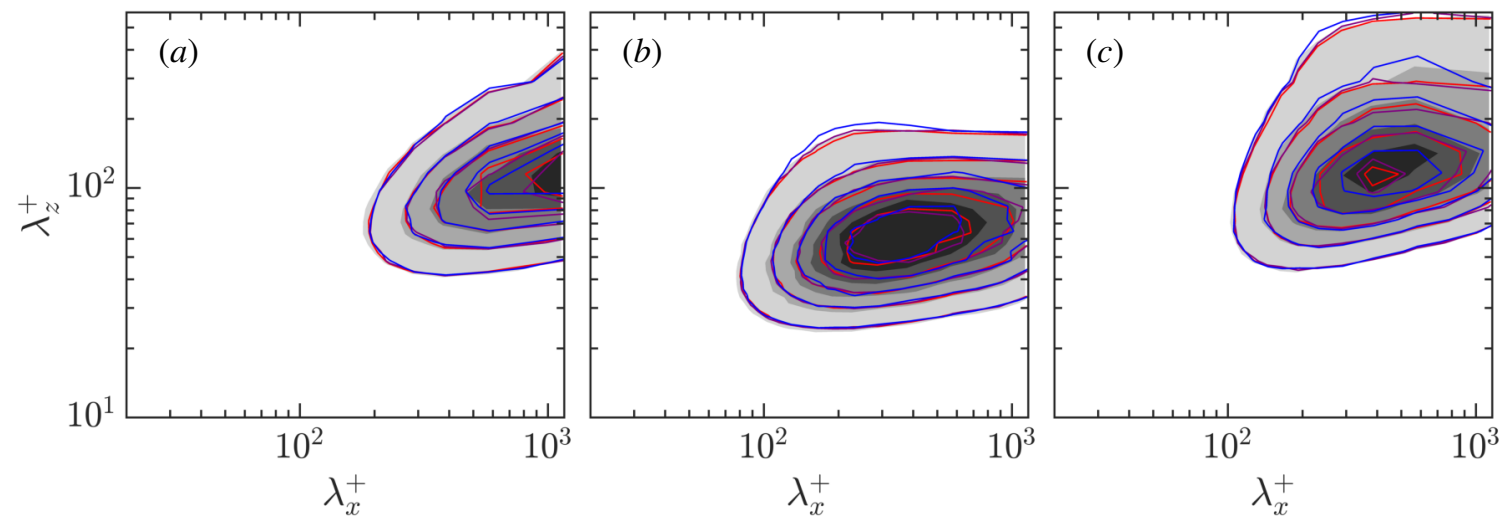

Figure 11: Premultiplied two-dimensional spectral densities of $u^{2}, v^{2}$ and $w^{2}$. Shaded contours correspond to the smooth channel at $y^{+} \approx 10$ and the solid lines to the present DNSs shifted by their corresponding virtual origin of turbulence, i.e. at $y^{+} \approx 10-\ell_{T}^{+}$, and scaled with $u_{\tau}$ measured at $y^{+}=-\ell_{T}^{+}$. - DHV222; —, DHV333; —, DHV444. (a) $k_{x} k_{z} E_{u u}$, (b) $k_{x} k_{z} E_{v v} ;$ (c) $k_{x} k_{z} E_{w w}$. The contour increments in wall units are $0.3241,0.0092$ and 0.0404 , respectively.

(a)

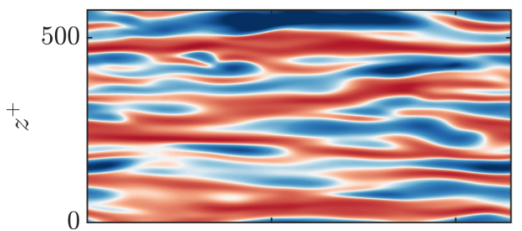

(b)

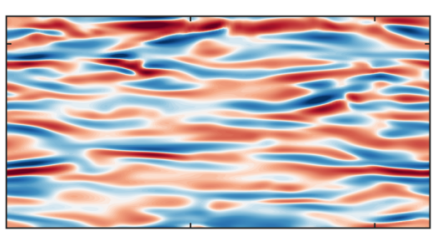

(c)

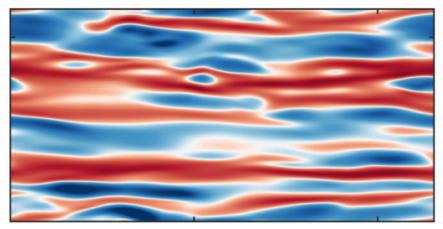

$(d)$

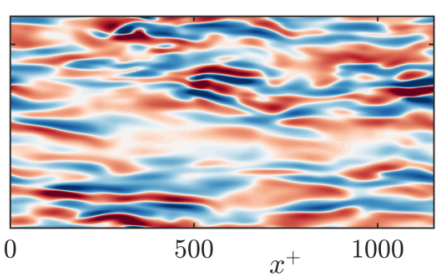

$(e)$

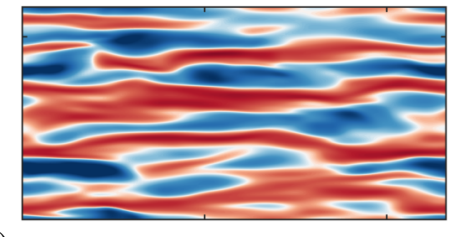

$(f)$

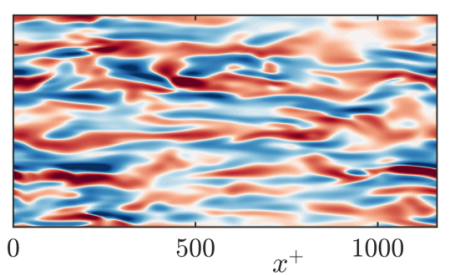

Figure 12: Instantaneous realisations of streamwise, $u^{\prime+}$, (top) and wall-normal, $v^{+}$, (bottom) velocities at a plane parallel to the wall. (a-b) Case DHV222 at $y^{+} \approx 7-\ell_{T}^{+} \approx 5.3$. (c-d) Case DHV333 at $y^{+} \approx 7-\ell_{T}^{+} \approx 4.7$. (e-f) Case DHV444 at $y^{+} \approx 7-\ell_{T}^{+} \approx 4.1$. In all cases, red to blue corresponds to $[-5.0,+5.0]$ for $u^{\prime+}$ and $[-0.48,+0.48]$ for $v^{\prime+}$.

are referred to as DHV220, DHV330 and DHV440, respectively, where, as before, the three digits correspond to the depths of the virtual origins imposed for $u, w$ and $v$; although due to the curvature effect of $w$, its origin is shallower than $y^{+}=-2,-3$ and -4 , respectively, as previously discussed and indicated in table 2 .

As mentioned before, the displacement of the quasistreamwise vortices towards the reference plane is determined by the origins of the spanwise and wall-normal velocities, which are set at $y^{+}=-\ell_{w}^{+}$and $y^{+}=-\ell_{v}^{+}=0$, respectively. The vortices approach the wall, as they are allowed to slip in the spanwise direction, but their downward displacement is now restricted by $v$ being zero at the simulation boundary. The origin that the vortices perceive, $y^{+}=-\ell_{T}^{+}$, is therefore at an intermediate height between $y^{+}=-\ell_{w}^{+}$and $y^{+}=-\ell_{v}^{+}$, that is, $\ell_{w}^{+}<\ell_{T}^{+}<\ell_{v}^{+}$. From the displacement of the profiles of $\omega_{x}^{\prime+}$ and ${\overline{u^{\prime} v^{\prime}}}^{+}$in figure 14, this origin is at a depth below the reference plane of $\ell_{T}^{+} \approx 1.3$ for case DHV220, $\ell_{T}^{+} \approx 1.7$ for case DHV330 and $\ell_{T}^{+} \approx 1.9$ for DHV440. This reflects the saturation effect of the spanwise slip discussed in section 1 . Notice that the $v^{\prime+}$ profiles in panel 14(a.2) are also shifted in $y^{+}$relative to the smooth-wall profile, even though the boundary condition remains $v^{+}=0$ at the reference plane. This is because $u^{+}$and $w^{+}$are not zero at this plane, and from continuity, the gradient $d v^{+} / d y^{+}$is no longer zero. This results in a shift in $y^{+}$of the $v^{\prime+}$-profiles, which coincides with $\ell_{T}^{+}$. The resulting virtual origin perceived by $v$ is therefore not at $y^{+}=0$, but at $y^{+}=-\ell_{T}^{+}$.

Fairhall and García-Mayoral (2018) proposed that the 


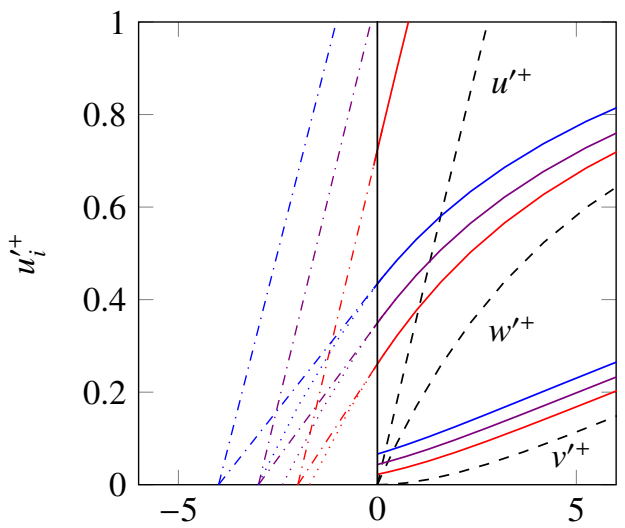

Figure 13: Rms velocity fluctuations near the reference plane. Zoom of the near-wall region from figure $10($ a.2). - - - , smooth channel; - , DHV222; — , DHV333; — _ DHV444. The dashed-dotted lines show the linear extrapolation of the profiles, which for the spanwise velocity vanish at $y^{+}=-\ell_{z}^{+}$, giving the spanwise slip length; and the dotted lines show the extrapolation retaining the curvature of smooth-wall profiles, which vanish at the virtual origin $y^{+}=-\ell_{w}^{+}$.

saturation effect in slip-only simulations could be represented by an empirical, effective spanwise slip length, $\ell_{z, \text { eff }}^{+}=\ell_{z}^{+} /\left(1+\ell_{z}^{+} / 4\right)$. However, given that the curvature of $w^{\prime+}$ needs to be accounted for, it would be more appropriate to express the prediction of $\ell_{T}^{+}$in terms of $\ell_{w}^{+}$instead of $\ell_{z}^{+}$, which yields

$$
\ell_{w, e f f}^{+}=\frac{\ell_{w}^{+}}{1+\ell_{w}^{+} / 4}
$$

The good agreement between the values of $\ell_{T}^{+}$measured a posteriori in the DNSs and those predicted using equation (6), $\ell_{T, \text { pred }}^{+}=\ell_{w, \text { eff }}^{+}$, can be observed in table 2 . Equation (6) provides therefore an expression to predict $\ell_{T}^{+}$when $\ell_{v}^{+}=0$.

The present results agree with the observations from the previous section 3.1.4, and support the idea that the near-wall cycle is shifted in block by $\ell_{T}^{+}$, but remains otherwise smooth-wall-like. This is evidenced by the excellent collapse with the smooth-wall data observed in the one-point statistics and the spectral density distributions in figures $14(b .1-b .4)$ and 15 , where they have all been shifted by $\ell_{T}^{+}$, with $u_{\tau}$ measured at that origin. This implies that the effect of virtual origins on the overlying turbulence is represented well by a single parameter, the origin of turbulence, $\ell_{T}^{+}$, although there are small differences immediately above the reference plane, caused by the specific boundary conditions imposed. The profiles of $u^{\prime+}$ in figure 14(b.2), for instance, decay linearly towards the streamwise virtual origin, $y^{+}=-\ell_{x}^{+}$, which is deeper than $y^{+}=-\ell_{T}^{+}$. The viscous sublayer is therefore thickened, modifying the gradient in the proximity of $y^{+}=0$ and resulting also in a slight increase of the magnitude of the peak. This, however, does not appear to alter the near-wall dynamics.

From the present results, the imposed virtual origins $\ell_{u}^{+}, \ell_{v}^{+}$and $\ell_{w}^{+}$(or alternatively the slip lengths $\ell_{x}^{+}, \ell_{y}^{+}$ and $\ell_{z}^{+}$) appear to have separate effects on the overlying flow. The latter two set the origin of turbulence, $\ell_{T}^{+}$, and hence determine the displacement of the near-wall cycle, while $\ell_{u}^{+}$is essentially inactive on modifying the turbulence cycle, and acts on determining the shift of the mean flow. This is illustrated by the mean velocity profiles shown in panel (a.1), where the origin of the mean flow is at a depth of 2, 3 and 4 wall units below the reference plane for the cases DHV220, DHV330 and DHV440, respectively, and hence $\ell_{U}^{+}=\ell_{u}^{+}$(where $\ell_{u}^{+}=$ $\left.\ell_{x}^{+}\right)$.

Taking the virtual origin of turbulence, $y^{+}=-\ell_{T}^{+}$, as the origin for the wall-normal coordinate, as in figure $14(b .1)$ and $10(b .1)$, shows that the difference between the mean velocity profiles reduces to an offset $\ell_{U}^{+}-\ell_{T}^{+}$, homogeneous along the entire $y$-range, and which corresponds to the value of $U^{+}$measured at the origin of turbulence. Luchini's linear law, given by equation (1), can then be generalised to

$$
\Delta U^{+}=\ell_{U}^{+}-\ell_{T}^{+}
$$

This equation supports the existing understanding that the change in drag is caused by the difference between the virtual origins perceived by the mean flow and that perceived by the overlying turbulence. In general, we can expect $\ell_{U}^{+} \approx \ell_{x}^{+}$, because in the viscous sublayer the mean velocity profile is essentially linear, which means that $\ell_{U}^{+}$can be predicted by $\ell_{x}^{+}$. However, the origin for turbulence, determined by the displacement of quasistreamwise vortices to the reference plane, is not fully defined by $\ell_{z}^{+}$alone, as originally postulated by Jiménez (1994) and Luchini (1996), but by a combination of the origins perceived by the spanwise and wall-normal velocities, $\ell_{w}^{+}$and $\ell_{v}^{+}$. This implies that drag reduction can still be obtained despite the slip lengths in the streamwise and spanwise directions being equal, as reported in the literature (Min and Kim, 2004; Busse and Sandham, 2012) and in contradiction with Luchini's original theory (1), due to the wall-normal velocity having a shallower virtual origin. Our preliminary results suggest that we could predict $\ell_{T}^{+}$from the spanwise and wall-normal virtual origins imposed a priori, $\ell_{w}^{+}$and $\ell_{v}^{+}$, or, alternatively from their corresponding slip lengths $\ell_{z}^{+}$ and $\ell_{y}^{+}$, as there is a one-to-one relationship between slip 

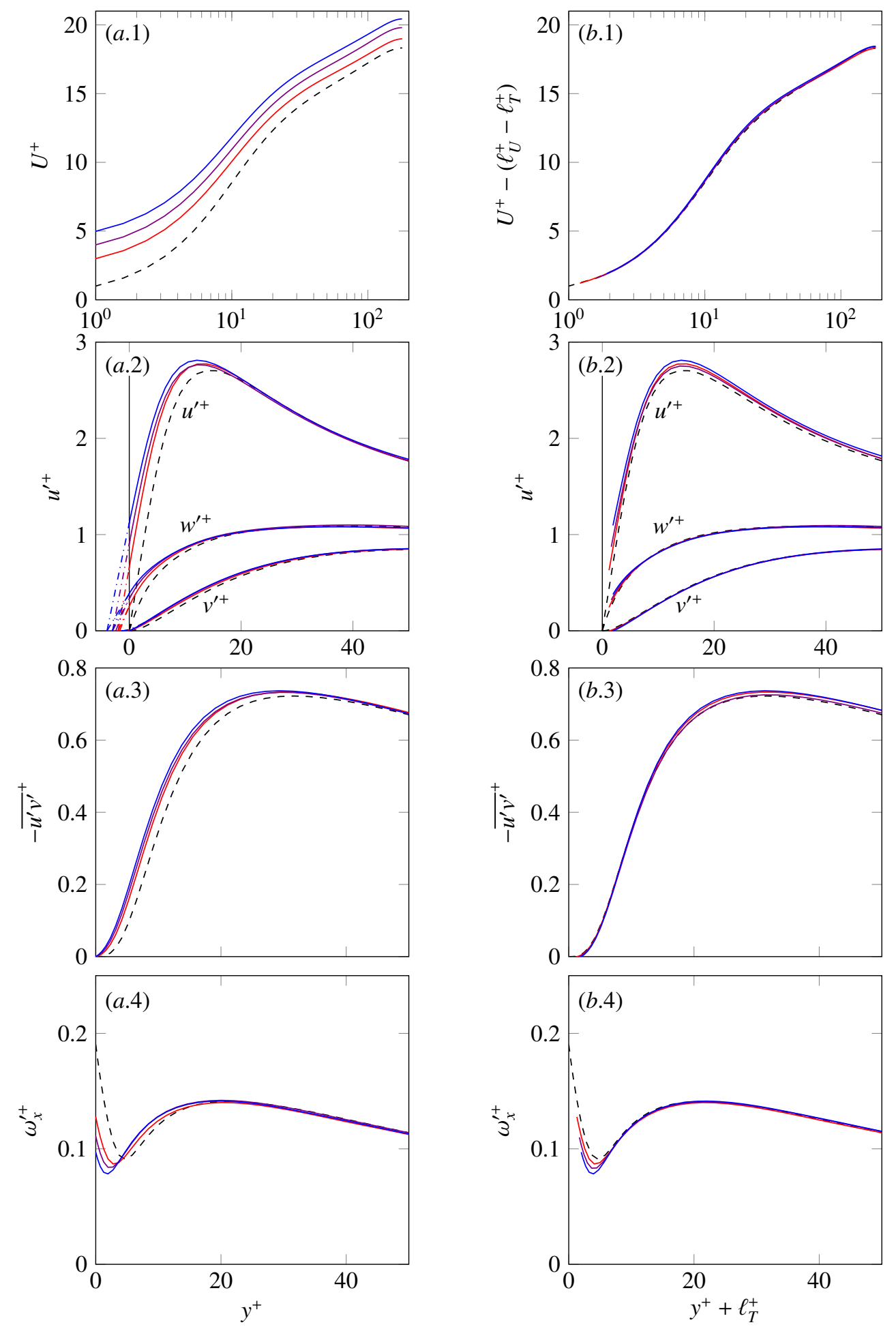

Figure 14: First column (a.1-a.4), profiles scaled with $u_{\tau}$ measured at the reference plane where the boundary conditions are imposed, $y^{+}=0$. Second column (b.1-b.4), profiles scaled with $u_{\tau}$ measured at the origin of turbulence, $y^{+}=-\ell_{T}^{+}$, and shifted by $\ell_{T}^{+}$. -- - , smooth channel; - , DHV220; — DHV330; —_ DHV440. ( $(a .1, b .1)$ Mean velocity profiles; $(a .2, b .2)$ rms velocity fluctuations; $(a .3, b .3)$ Reynolds shear stresses; $(a .4, b .4)$ streamwise vorticity fluctuations. 

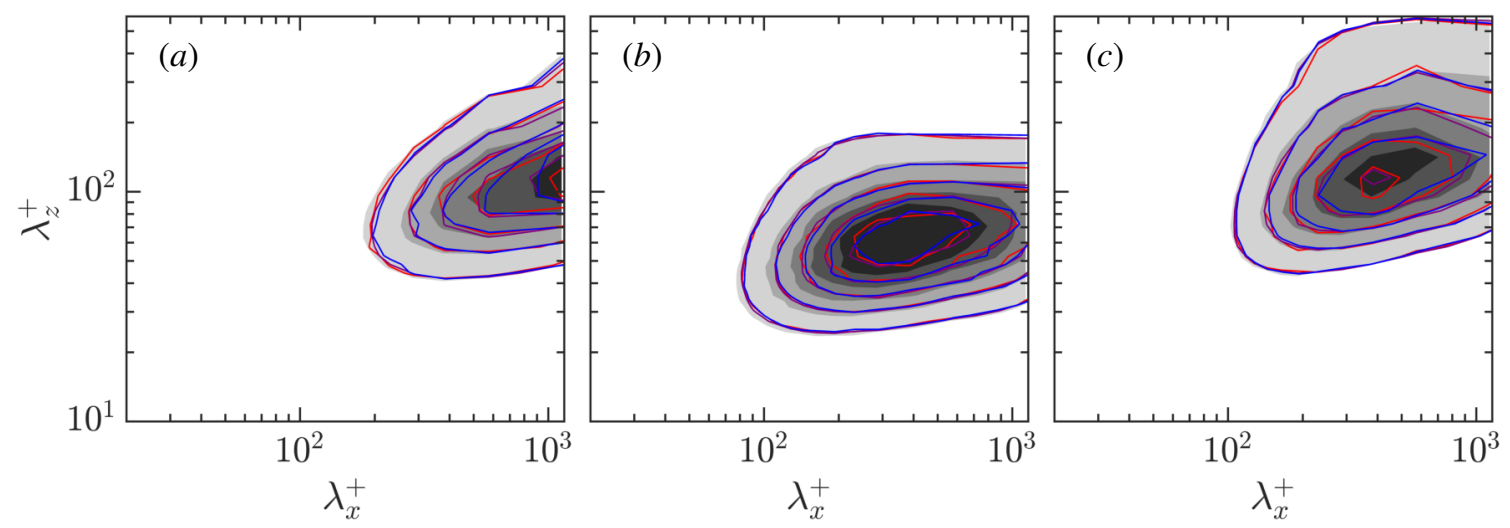

Figure 15: Premultiplied two-dimensional spectral densities of $u^{2}, v^{2}$ and $w^{2}$. Shaded contours correspond to the smooth channel at $y^{+} \approx 10$ and the solid lines to the present DNSs shifted by their corresponding virtual origin of turbulence, i.e. at $y^{+} \approx 10-\ell_{T}^{+}$, and scaled with $u_{\tau}$ measured at $y^{+}=-\ell_{T}^{+}$. - DHV220; — DHV330; — DHV440. (a) $k_{x} k_{z} E_{u u}$, (b) $k_{x} k_{z} E_{v v} ;$, (c) $k_{x} k_{z} E_{w w}$. The contour increments in wall units are $0.3241,0.0092$ and 0.0404 , respectively.

lengths and virtual origins. Knowing the slip lengths, $\ell_{x}^{+}, \ell_{z}^{+}$and $\ell_{y}^{+}$, the depths of the virtual origins, $\ell_{u}^{+}, \ell_{w}^{+}$ and $\ell_{v}^{+}$, can be obtained, and vice-versa. More simulations, covering a wider range of combinations of $\ell_{u}^{+}, \ell_{v}^{+}$ and $\ell_{w}^{+}$, are however required to derive a formal expression for $\ell_{T}^{+}$.

\section{Conclusions}

In flows over complex surfaces with small texture sizes, the velocity components may appear to vanish at different heights, that is, different virtual origins can be defined for the different velocity components. In the present work, we have explored different techniques to define and implement these virtual origins into DNSs, with special emphasis on the boundary conditions for the wall-normal velocity. We investigated impedance conditions derived from a Stokes-flow model, which relate the wall-normal velocity to the pressure, as well as Robin conditions for the three velocities, which relate velocity components with their wall-normal gradients. We have shown that Robin boundary conditions on the three velocities, as given by equation (5), can effectively capture the effect of virtual origins on the overlying flow, and provide a first step towards the formulation of simplified models for textured surfaces.

Our results suggest that the effect of the three virtual origins on the overlying flow can be reduced to a different virtual origin perceived by the mean flow, $y^{+}=-\ell_{U}^{+}$, and by the overlying turbulence, $y^{+}=-\ell_{T}^{+}$, confirming Luchini's original idea. The origin of turbulence, however, is not determined by the origin of the spanwise velocity alone, but by the origins perceived by the spanwise and wall-normal velocities. Therefore, other than a shift in the wall-normal coordinate by $\ell_{T}^{+}$, the near-wall turbulence and its dynamics remain smooth-wall-like. By this shift, we implicitly refer not only to a displacement in $y^{+}$by $\ell_{T}^{+}$, but also to a rescaling of the results by the friction velocity defined at $y^{+}=-\ell_{T}^{+}$. As a result, the only change in the mean velocity profile is an offset by its value at the origin for turbulence, $\Delta U^{+}=\ell_{U}^{+}-\ell_{T}^{+}$, which generalises Luchini's linear theory for the change of drag.

There are, however, two main limitations to the model of Robin boundary conditions. First, the virtualorigin model implicitly assumes that the near-wall cycle remains canonical, that is, it remains as that over a smooth wall, and hence the imposed virtual origins can only be of a few wall units, $\ell^{+} \simeq 10$, so that the near-wall cycle is not completely ingested below the reference plane. Second, the validity of Robin boundary conditions relies on the velocity rms profiles being smooth-wall-like independently from each other, since the slip lengths are obtained based on the wall-normal gradients of the smooth profiles. This implies that the present model and the expression for $\ell_{T}^{+}$are valid as long as the virtual origins set for the three velocity components differ only by a few wall units between them. If the origins perceived by the different components differ substantially, the resulting wall-normal gradients at the reference plane may be modified from those of a smooth wall, due to the coupling between the velocities just above the reference plane. As a result, the actual origins would be different to those expected. Additionally, assuming that these Robin boundary conditions 
are applicable to model the effect of real complex surfaces, there is the additional effect of the texture granularity that the model is not able to capture (Fairhall et al., 2019). Further investigation is therefore needed to find if the virtual-origin model developed here can adequately account for the change in drag produced by different complex surfaces.

\section{Acknowledgements}

This work was started as part of the 3rd Madrid Summer Workshop on Turbulence, funded by the ERC Coturb project, grant ERC-2014.AdG-66950. The work was also funded by EPSRC UK under grant EP/S013083/1. GG was partially supported by an educational grant from Fundación Bancaria 'la Caixa'. Some of the computational resources were provided by the Cambridge Service for Data Driven Discovery under EPSRC grant EP/P020259/1.

\section{References}

Bottaro, A., 2019. Flow over natural or engineered surfaces: an adjoint homogenization perspective. J. Fluid Mech. 877, P1.

Bottaro, A., Naqvi, S., 2020. Effective boundary conditions at a rough wall: a high-order homogenization approach. Submitted to Meccanica .

Busse, A., Sandham, N.D., 2012. Influence of an anisotropic sliplength boundary condition on turbulent channel flow. Phys. Fluids 24, 055111.

Choi, H., Moin, P., Kim, J., 1994. Active turbulence control for drag reduction in wall-bounded flows. J. Fluid Mech. 262, 75-110.

Chung, D., Hutchins, N., Schultz, M.P., Flack, K.A., 2021. Predicting the Drag of Rough Surfaces. Annu. Rev. Fluid Mech. 53, 1-35.

Clauser, F.H., 1956. The turbulent boundary layer. Adv. App. Mech. 4, 1-51

Encinar, M.P., García-Mayoral, R., Jiménez, J., 2014. Scaling of velocity fluctuations in off-wall boundary conditions for turbulent flows. J. Phys. Conf. Ser. 506, 012002.

Fairhall, C.T., Abderrahaman-Elena, N., García-Mayoral, R., 2019. The effect of slip and surface texture on turbulence over superhydrophobic surfaces. J. Fluid Mech. 861, 88-118.

Fairhall, C.T., García-Mayoral, R., 2018. Spectral analysis of sliplength model for turbulence over textured superhydrophobic surfaces. Flow Turb. Combust. 100, 961-978.

Fukagata, K., Kasagi, N., Koumoutsakos, P., 2006. A theoretical prediction of friction drag reduction in turbulent flow by superhydrophobic surfaces. Phys. Fluids 18, 051703.

García-Mayoral, R., Gómez-de-Segura, G., Fairhall, C.T., 2019. The control of near-wall turbulence through surface texturing. Fluid Dyn. Res. 51, 011410.

García-Mayoral, R., Jiménez, J., 2011. Drag reduction by riblets. Phil. Trans. R. Soc. A 369, 1412-1427.

García-Mayoral, R., Jiménez, J., 2011. Hydrodynamic stability and breakdown of the viscous regime over riblets. J. Fluid Mech. 678, 317-347.

Gatti, D., Quadrio, M., 2016. Reynolds-number dependence of turbulent skin-friction drag reduction induced by spanwise forcing. J. Fluid Mech. 802, 553-582.
Gómez-de-Segura, G., 2019. Turbulent drag reduction by anisotropic permeable substrates. Ph.D. thesis. University of Cambridge.

Gómez-de-Segura, G., Fairhall, C.T., MacDonald, M., Chung, D., García-Mayoral, R., 2018. Manipulation of near-wall turbulence by surface slip and permeability. J. Phys. Conf. Ser. 1001, 012011.

Gómez-de-Segura, G., García-Mayoral, R., 2019. Turbulent drag reduction by anisotropic permeable substrates - analysis and direct numerical simulations. J. Fluid Mech. 875, 124-172.

Jiménez, J., 1994. On the structure and control of near wall turbulence. Phys. Fluids 6, 944.

Jiménez, J., 2004. Turbulent flows over rough walls. Annu. Rev. Fluid Mech. 36, 173-196.

Jiménez, J., Uhlmann, M., Pinelli, A., Kawahara, G., 2001. Turbulent shear flow over active and passive porous surfaces. J. Fluid Mech. 442, 89-117.

Kamrin, K., Bazant, M.Z., Stone, 2010. Effective slip boundary conditions for arbitrary periodic surfaces: The surface mobility tensor. J. Fluid Mech. 658, 409-437.

Lācis, U., Sudhakar, Y., Pasche, S., Bagheri, S., 2020. Transfer of mass and momentum at rough and porous surfaces. J. Fluid Mech. 884, A 21 .

Lauga, E., Stone, H.A., 2003. Effective slip in pressure-driven Stokes flow. J. Fluid Mech. 489, 55-77.

Le, H., Moin, P., 1991. An improvement of fractional step methods for the incompressible Navier-Stokes equations. J. Comput. Phys. 92, 369-379.

Lozano-Durán, A., Jiménez, J., 2014. Effect of the computational domain on direct numerical simulations of turbulent channels up to $R e_{\tau}=4200$. Phys. Fluids 26, 011702 .

Luchini, P., 1996. Reducing the turbulent skin friction. Comput. Methods Appl. Sci. - Proc. 3rd ECCOMAS CFD Conf. , 466-470.

Luchini, P., 2013. Linearized no-slip boundary conditions at a rough surface. J. Fluid Mech. 737, 349-367.

Luchini, P., Manzo, F., Pozzi, A., 1991. Resistance of a grooved surface to parallel flow and cross-flow. J. Fluid Mech. 228, 87-109.

Min, T., Kim, J., 2004. Effects of hydrophobic surface on skin-friction drag. Phys. Fluids 16, L55.

Mizuno, Y., Jiménez, J., 2013. Wall turbulence without walls. J. Fluid Mech. 723, 429-455.

Orlandi, P., Jiménez, J., 1994. On the generation of turbulent wall friction. Phys. Fluids 6, 634-641.

Perot, J.B., 1993. An analysis of the fractional step method. J. Comput. Phys. 108, 51-58.

Philip, J.R., 1972. Flows Satisfying Mixed No-Slip and No-Shear Conditions. Angew. Math. Phys. 23, 353-372.

Robinson, S.K., 1991. Coherent motions in the turbulent boundary layer. Annu. Rev. Fluid Mech. 23, 601-639.

Rothstein, J.P., 2010. Slip on superhydrophobic surfaces. Annu. Rev. Fluid Mech. 42, 89-109.

Schoppa, W., Hussain, F., 2002. Coherent structure generation in nearwall turbulence. J. Fluid Mech. 453, 57-108.

Spalart, P.R., McLean, J.D., 2011. Drag reduction: enticing turbulence, and then an industry. Phil. Trans. R. Soc. A 369, 1556-1569.

Walsh, M.J., Lindemann, A.M., 1984. Optimization and application of riblets for turbulent drag reduction. AIAA Pap. 84, 0347. 